Poly (lactide-co-glycolide) Nanoparticles Mediate Sustained Gene Silencing and Improved

Lan Wu ${ }^{1}$, Lin-Ping Wu $u^{2}$, Jingya Wu ${ }^{1}$, Jin Sun ${ }^{1}$, Zhonggui He ${ }^{l}$, Cristina Rodriguez-Rodriguez ${ }^{3,4}$, Katayoun Saatchi ${ }^{3}$, Lea Ann Dailey ${ }^{5}$, Urs O. Häfeli ${ }^{3,6}$, Dongmei Cun ${ }^{1 *}$, Mingshi Yang ${ }^{1,6^{* *}}$

3: Faculty of Pharmaceutical Sciences, University of British Columbia, 2405 Wesbrook Mall, Vancouver, BC, V6T 1Z3, Canada

4: Department of Physics \& Astronomy, University of British Columbia, 6224 Agricultural Road, Vancouver, BC V6T 1Z1, Canada

5: Department of Pharmaceutical Technology and Biopharmaceutics, University of Vienna, Althanstraße 14 (UZA II), 1090 Vienna, Austria

6: Department of Pharmacy, Faculty of Health and Medical Sciences, University of Copenhagen, Universitetsparken 2, 2100 Copenhagen, Denmark

* To whom correspondence should be addressed: Mingshi Yang, mingshi.yang@sund.ku.dk; Dongmei Cun, cundongmei@163.com. 


\section{A. METHODS}

25 1. Validation of the Method for Determining the Encapsulation Efficiency (EE) of siRNA in the 26 LPXs

27 2. Investigation of the In Vitro Release of siRNA From the LPXs and LPNs

28 3. Establishment of A549-EGFP Cell Line

29 4. Effect of Anionic Endogenous Agent Hyaluronic Acid (HA) on Gene Silencing Performances of 30 LPNs and LPXs

31 5. Colloidal Stability Test of LPNs and LPXs

32 6. In Vitro Cell Uptake Studies of LPNs and LPXs

33 7. Genotyping of EGFP Transgenic Mice

34 8. Comparation of Transgenic Mice Tissue Fluorescence and Wild Type Mice Tissue 35 Autofluorescence

36 9. Verification of the Linear Correlation Between Total Protein Concentration and Fluorescence 37 Intensity of EGFP in Lung Tissues

38 10. Flow Cytometry Analysis for In Vitro Gene Silencing Efficiency and Cell Viability Assays

39 11. Radiolabeling Procedure, Radiolabeling Efficiency Test and Particle Properties Change After $40 \quad$ Radiolabeling

41 12. SPECT/CT Scanning Condition and Reconstruction

42 13. Decay Correction

43 14. Calculation of Radioactivity in 3D volume of interest (VOIs) from Single Photon Emission 44 Computed Tomography/Computed Tomography (SPECT/CT) Images 
45 15. Calculation of Gamma Counting

46 16. Endotoxin Test

47 17. Determination of Cytokine Levels and Total Protein Level in BALF

\section{B. RESULTS AND DISCUSSION}

49

1. Validation of the Method for the Determination of Encapsulation Efficiency (EE) of siRNA in 50 LPXs

51 2. Investigation of the In Vitro Release of siRNA from LPXs and LPNs

52 3. Effect of Anionic Endogenous Agent Hyaluronic Acid (HA) on Gene Silencing Performances of $53 \quad$ LPNs and LPXs

54 4. Colloidal Stability Test of LPNs and LPXs

55 5. In Vitro Cell Uptake Studies of LPNs and LPXs

56 6. Genotyping of EGFP Transgenic Mice

57 7. Comparation of Transgenic Mice Tissue Fluorescence and Wild Type Mice Tissue 58 Autofluorescence

59 8. Verification of the Linear Correlation Between Total Protein Concentration and Fluorescence 60 Intensity of EGFP in Lung Tissues

61 9. Flow Cytometry Analysis for In Vitro Gene Silencing Efficiency and Cell Viability Assays

62 10. Western Blotting and CLSM Images of Lung Tissue

63 11. Radiolabeling Efficiency and Particle Properties Change Before and After Radiolabeling

64 12. Endotoxin test

65 13. Cytokine Levels in BALF

66 A. METHODS 
In order to validate the method for the determination of EE of siRNA in LPXs, a spiking experiment 49.6 $\mu \mathrm{M}$ siRNA solution to prepare the LPXs. The LPXs suspension was then diluted with RNase-free TE buffer to acquire the final concentration of siRNA of $100 \mathrm{nM}$. Subsequently, $50 \mu \mathrm{L}$ of the diluted LPXs suspension was placed into each well of a 96-wells plate, which was further diluted with $50 \mu \mathrm{L}$ of HD solution or TE buffer, respectively. After incubated at a room temperature for 30 min, $100 \mu \mathrm{L}$ of RiboGreen ${ }^{\circledR}$ RNA reagent was added, and the concentrations of siRNA in the spiked samples with or without the addition of HD solution were measured using a fluorescent microplate reader, respectively. The recovery of siRNA was expressed as the percentage of measured siRNA concentration relative to the original concentration.

\section{Investigation of the In Vitro Release of siRNA From LPXs and LPNs}

The in vitro release profile of siRNA from LPNs was investigated by following the previous procedure with minor modification. ${ }^{1}$ In brief, $3.75 \mathrm{mg}$ of LPNs with a N/P ratio of 10 was dispersed in $300 \mu \mathrm{L}$ of $\mathrm{pH} 7.4 \mathrm{RNase}$-free TE buffer (10 mM Tris, $100 \mathrm{mM} \mathrm{NaCl,} 1 \mathrm{mM}$ EDTA) and incubated in a $37.0 \pm 0.5^{\circ} \mathrm{C}$ incubator and horizontally shaken at $50 \mathrm{rpm}$. At each predetermined time point, the release medium was collected after centrifuging the LPNs dispersion at $13,600 \times \mathrm{g}$ for 10 min and replaced with equal volume of fresh TE buffer. The concentration of free siRNA in the release medium was measured using RiboGreen ${ }^{\circledR}$ RNA reagent kit without adding of HD, while the concentration of total siRNA (free siRNA plus bonded siRNA) was measured by using the same RiboGreen ${ }^{\circledR}$ RNA reagent kit but with an addition of HD solution to disassociate siRNA from bounded form as mentioned in section 2.3.

The release of the LPXs was conducted using LPXs suspension with a N/P ratio of 10 and the final 
91 siRNA concentration of $100 \mathrm{nM}$. The LPXs suspension was placed in a plain bottom 96-wells plate followed by an addition of $200 \mu \mathrm{L}$ of LPXs suspension into each well. The plate was sealed with parafilm ${ }^{\circledR}$ and incubated at $37.0 \pm 0.5^{\circ} \mathrm{C}$. At each predetermined time point, the samples from 3 different wells were withdrawn for siRNA concentration determination with or without the addition of HD solution.

\section{Establishment of A549-EGFP Cell Line}

The A549-EGFP cell line was purchased from Obio Technology Corp., Ltd. Shanghai, China. All the procedures of cell line establishment were provided by Obio Technology Corp. Lentiviral vectors containing EGFP and puromycin resistance gene was generated by Obio Technology Corp., Ltd. Shanghai, China.

A549 cells were seeded into 24-well tissue culture plates (Corning Co. Ltd, NY, USA) at a density of $7 \times 10^{4}$ cells/well. After $24 \mathrm{~h}$ of growth ( $30 \%$ confluence), the culture medium was replaced with 1 $\mathrm{mL}$ of fresh medium containing lentiviral vectors at a multiplicity of infection (MOI) of 40 with the presence of $10 \mu \mathrm{g} / \mathrm{mL}$ of polybrene (Sigma-Aldrich, St. Louis, MO, USA). After $8 \mathrm{~h}$ of incubation, the medium was replaced with $500 \mu \mathrm{L}$ of fresh medium. After another $72 \mathrm{~h}$ of incubation, the medium was replaced with $1 \mathrm{~mL}$ of medium containing $2 \mu \mathrm{g} / \mathrm{mL}$ of puromycin. The cells were performed with regular passage and the growth of cells were monitored. Once the living cells grew well, the expression of EGFP protein was checked by fluorescence microscope and the cells were stocked.

\section{Effect of Anionic Endogenous Agent Hyaluronic Acid (HA) on Gene Silencing Performances of LPNs and LPXs}

The gene silencing performances of LPXs or LPNs with the challenge of endogenous agents was conducted with HA as a model endogenous agent in the lung. The LPXs or LPNs with a N/P ratio of 10 were dispersed in mediums containing $0.1 \%, 0.3 \%$ or $0.5 \%$ of $\mathrm{HA}(\mathrm{w} / \mathrm{v})$, which were further diluted with serum-free F-12K cell culture medium in an appropriate ratio to achieve $40 \mathrm{nM}$ or 80 
$\mathrm{nM}$ of siRNA concentration. One milliliter of the sample was then added into 24-well plates pre-seeded with A549-EGFP cells (initial density of $6 \times 10^{4}$ cells/well, 30\% confluence). After incubating for $8 \mathrm{~h}$, the medium was removed and the cells were washed twice with $1 \mathrm{~mL}$ of $\mathrm{pH} 7.4$ phosphate-buffered saline (PBS, Sigma-Aldrich) and further cultured in $1 \mathrm{~mL}$ full medium for another $40 \mathrm{~h}$. The cells were then collected for flow cytometry according to the procedures described in section 2.5. The A549-EGFP cells were also seeded into 6 well plates with an initial density of $2 \times$ $10^{5}$ cells/well, treated with the same method above and collected for Western blotting. The cells were lysed with $80 \mu \mathrm{L}$ RIPA buffer added with phenylmethylsulfonyl fluoride (PMSF) and protease inhibitor at $4{ }^{\circ} \mathrm{C}$ for $1.5 \mathrm{~h}$. The mixture was then centrifuged at $14000 \times \mathrm{g}$ for $15 \mathrm{~min}$. The supernatant was collected and the protein level of supernatant was measured using BCA method. The supernatant containing $20 \mu \mathrm{g}$ protein was mixed with equal volume of loading buffer and separated on $12 \%$ SDS-PAGE gels. The protein was transferred to a nitrocellulose membrane. The membrane was blocked with $5 \%$ nonfat dry milk and then incubated with primary antibody to EGFP (clone differences between groups.

\section{Colloidal Stability Test of LPNs and LPXs}

The LPNs and LPXs with an N/P ratio of 10 and a siRNA concentration of $0.3 \mu \mathrm{M}$ were dispersed in a simulated lung fluid (SLF) prepared according to the composition reported in literature. ${ }^{2}$ The size, 
141 PDI, and zeta potential of LPNs and LPXs were measured after $1 \mathrm{~h}$ and $24 \mathrm{~h}$ of the incubation in this SLF.

\section{In Vitro Cell Uptake Studies of LPNs and LPXs}

The A549-EGFP cells were seeded into 6-well plates at a density of $3 \times 10^{5}$ cells/well. After growing overnight, the cells were incubated with Cy3-siRNA loaded LPNs or LPXs with N/P ratio of 10 and siRNA concentration of $50 \mathrm{nM}$ for $4 \mathrm{~h}$. The cells were then collected and the fluorescence intensity of Cy3 was analyzed using a cytometer (BD FACSCalibur, BD bioscience, USA).

For CLSM imaging, the A549-EGFP cells were grown on coverslips in 6-well plates at a density of $3 \times 10^{5}$ cells/well and incubated with Cy3-siRNA loaded LPNs or LPXs as described above. After incubating for $4 \mathrm{~h}$, the cells were washed three times using PBS and fixed for 30 min with $4 \%$ formaldehyde at room temperature. The cells were then washed three times using PBS and the nuclei was stained with DAPI $(10 \mu \mathrm{g} / \mathrm{mL}$, Invitrogen Ltd. Paisley, UK). The samples were then mounted onto the slides and observed using CLSM.

\section{Genotyping of EGFP Transgenic Mice}

The C57BL/6-Tg (CAG-EGFP) mouse line is a well-established mouse line from the Jackson's lab with widespread EGFP fluorescence with the exception of erythrocytes and hair (https://www.jax.org/strain/003291). Since all the somatic cells of a living C57BL/6-Tg (CAG-EGFP) mouse have the same gene composition, a small piece of tissue from the tip of mouse tails was reasonably used as the representative sample for genotyping.

The total DNA from the tail tip of 45 purchased EGFP transgenic mice and 3 wild type (WT) mice was extracted using a DNA extraction kit (DP 1902, Bioteke Corporation, Beijing, China) according to the manufacturer's instruction. In brief, a single tail tip (Ca. $0.5 \mathrm{~cm}$ in length) was placed into a mixture of $200 \mu \mathrm{L}$ of tissue lysis buffer and $20 \mu \mathrm{L}$ of protease $\mathrm{K}(20 \mathrm{mg} / \mathrm{mL})$, and incubated at $55^{\circ} \mathrm{C}$ for $3 \mathrm{~h}$. The system was then added with $200 \mu \mathrm{L}$ of combination buffer and $100 \mu \mathrm{L}$ of isopropanol 
and mixed up. After centrifugation at $13000 \mathrm{rpm}$ for $2 \mathrm{~min}$, the supernatant was transferred into a spin column and centrifuged at $10000 \mathrm{rpm}$ for $30 \mathrm{~s}$. The effluent was discarded, and $500 \mu \mathrm{L}$ of inhibitor remover was added into the spin column. The spin column was then centrifuged at 12000 rpm for $30 \mathrm{~s}$, and the effluent was discarded. Then $700 \mu \mathrm{L}$ of wash buffer was added onto the spin column and centrifuged at $12000 \mathrm{rpm}$ for $30 \mathrm{~s}$ to wash the spin column. After the washing procedure was repeated once, $80 \mu \mathrm{L}$ of elution buffer was added into the spin column and incubated for 5 min. incubated for another $2 \mathrm{~min}$ and centrifuged at $12000 \mathrm{rpm}$ for $1 \mathrm{~min}$. The collected effluent containing DNA was then used for standard polymerase chain reaction (PCR) for genotyping. $50 \mu \mathrm{L}$ of PCR reaction mix was prepared by mixing $10 \mu \mathrm{L}$ of mouse DNA (containing $0.4 \mu \mathrm{g}$ of DNA), $1 \mu \mathrm{L}$ of EGFP forward primer, $1 \mu \mathrm{L}$ of EGFP reverse primer, $13 \mu \mathrm{L}$ of $\mathrm{ddd}_{2} \mathrm{O}$ and $25 \mu \mathrm{L}$ of

GACCATGTGATCGCGCTTCTCGTT with sequence 5'-3'. The process of PCR reaction was: $94{ }^{\circ} \mathrm{C}, 2 \mathrm{~min} ; 94{ }^{\circ} \mathrm{C}, 30 \mathrm{~s}, 60{ }^{\circ} \mathrm{C}, 30 \mathrm{~s}, 72{ }^{\circ} \mathrm{C}, 30 \mathrm{~s}$ for 30 cycles; $72{ }^{\circ} \mathrm{C}, 2 \mathrm{~min}, 10{ }^{\circ} \mathrm{C}$, hold forever. The reacted mixture $(5 \mu \mathrm{L})$ was loaded to $2 \%$ agarose gel and conducted with electrophoresis at 130 $\mathrm{V}$ for $30 \mathrm{~min}$.

\section{Comparation of Transgenic Mice Tissue Fluorescence and Wild Type Mice Tissue}

\section{Autofluorescence}

The levels of EGFP fluorescent of the lung tissues and tissue lysate of a EGFP transgenic mouse and a WT mouse were detected using an IVIS imaging system (IVIS Lumina Series III, PerkinElmer, USA) in order to evaluate the influence of tissue autofluorescence on the EGFP fluorescence intensity. The lung tissues of a EGFP transgenic mouse and a WT mouse were put into a 6-well plate for comparison. The tissue lysate was either put in Eppendorf tubes for direct observation or added into a 96-well plate as done in the study. 
By following the procedure described in section 2.6.1, small pieces of lung tissue from EGFP transgenic mice were lysed and collected. The tissue lysate supernatant was then diluted 2.5, 5 and 25 times with RIPA buffer respectively. The fluorescence intensity and concentration of total protein in each diluted sample were measured. The linearity between the total protein level and fluorescence intensity was verified by linear fitting with software Origin 9.0.

\section{Flow Cytometry Analysis for In Vitro Gene Silencing Efficiency and Cell Viability Assays}

Flow cytometry analysis for in vitro gene silencing efficiency and cell viability assays was conducted using MACSQuantify software (Miltenyi Biotec). A number of 10,000 events was counted for each sample. Data of EGFP and PI fluorescence intensities were presented as the mean fluorescence intensity of FITC-A and PerCP-Vio700-A channels respectively. The gating strategies were kept constant for all samples.

\section{After Radiolabeling}

The radiolabeling of the LPNs and LPXs with ${ }^{111} \mathrm{In}$ was performed by adding $0.15 \mathrm{mg}$ of 1,2-distearoyl-sn-glycero-3-phosphoethanolamine-diethylenetriaminepentaacetic acid (PE-DTPA, Avanti Polar Lipids, Alabaster, AL, USA) to $2.10 \mathrm{mg}$ of DOTAP, and the mixture was used to prepare LPXs-DTPA and LPNs-DTPA with the procedure given in section 2.2. To a $100 \mu \mathrm{L}$ suspension of LPNs-DTPA or LPXs-DTPA (siRNA concentration: $12 \mu \mathrm{M}$ ) was added $44.4 \mathrm{MBq}$ ${ }^{111} \mathrm{InCl}_{3}(1.2 \mu \mathrm{L}$ of a $0.1 \mathrm{~N} \mathrm{HCl}$ solution) and incubated at room temperature for $30 \mathrm{~min}$ in a Thermomixer R (Eppendorf, Mississauga, Ontario, Canada) shaking at $500 \mathrm{rpm}$. The ${ }^{111} \mathrm{In}$ radiolabeled LPNs were then collected by centrifugation at $10,000 \times \mathrm{g}$ for $10 \mathrm{~min}$ and re-suspended 213 in distilled water. The ${ }^{111}$ In radiolabeled LPXs were directly used for the imaging experiments. Their 
radiolabeling efficiency and stability was tested by instant thin layer chromatography (ITLC) using 0.1 M EDTA as the mobile phase, developed on a photostimulable phosphor plate (Packard), scanned on a Cyclone Phosphorimager (Packard) and quantified by OptiQuant software (PerkinElmer,

217 Waltham, MA, USA). The particle size and zeta potential of LPNs and LPXs before and after being 218 radiolabeled with ${ }^{111} \mathrm{InCl}_{3}$ were measured with Malvern Zetasizer Nano ZS 90 by following the 219 procedure described in section 2.3.

\section{SPECT/CT Scanning Condition and Reconstruction}

221

$232 d c=e^{\left(-\frac{t}{t_{1} / 2} \times \ln 2\right)}$ Eq.S1

The decay factors at different time points were abbreviated as dc. The time from initial measuring of radioactivity was recorded accurately in minutes and referred to as t. The half-life of ${ }^{111}$ In $(4038.78$ min) was referred to as $\mathrm{t}_{1 / 2}$. 
The 3D VOIs were drawn manually based on registered CT images using AMIDE software (v.1.0.5, Stanford University, Stanford, CA, USA). The accuracy of 3D VOIs drawing was further verified by checking the SPECT images to make sure all the radioactivity was included in the VOIs. A calibration factor (CF) was determined by scanning the ${ }^{111}$ In-LPNs suspension and ${ }^{111}$ In-LPXs with known concentration of ${ }^{111}$ In.

The mean activity in each VOI (counts/pixel) and fraction voxels value (Frac. voxels) were 244 calculated by AMIDE. The calibration factor for ${ }^{111} \mathrm{In}$ was $882.93 \mathrm{MBq} / \mathrm{mL}$. The radioactivity of 245 each VOI was calculated according to equation S2 below.

\section{Radioactivity $=C F \times$ mean activity $\times$ Frac. voxels $\div$ dc $\quad$ Eq.S2}

\section{Calculation of Gamma Counting}

248 After euthanizing the mice at $11^{\text {th }}$ day after intratracheal administration. The blood, heart, liver, 249 kidneys, lungs, small intestine, brain, bladder, muscle, spleen, bone, pancreas, feces and stomach of 250 each mouse were collected, weighed and measured with radioactivity using a gamma counter 251 (Packard Cobra II autogamma counter, Perkin Elmer, Waltham, MA, USA) as described in section 252 2.7. The $\gamma$-counts per gram body weight $(\gamma$-counts/g) of each organ were calculated were calculated 253 according to equation S3 below.

$\gamma-$ counts $/ g=\frac{\gamma-\text { count }_{\text {organ }}}{\text { Weight }_{\text {organ }}} \quad$ Eq.S3

The biodistribution of nanoparticles in each organ was described by the percentage of radioactivity 
detected with in the organs relative to the total radioactivity in mice at $11^{\text {th }}$ day after intratracheal

257 administration. The percentage of organ biodistribution was calculated according to equation S4 258 below.

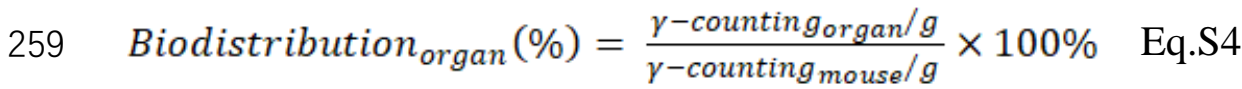

\section{Endotoxin test}

261 The LPNs and LPXs containing $0.3 \mathrm{nmol}(5 \mu \mathrm{g})$ of siRNA were dispersed in $100 \mu \mathrm{L}$ of Limulus 262 Amebocyte Lysate (LAL) reagent water and conducted with LAL assay using a LAL endotoxin assay 263 kit (Xiamen Bioendo Technology Co., Ltd., Xiamen, China) according to the manufacturer's 264 instruction.

\section{Determination of Cytokine Level in BALF}

The concentration of cytokines IL-1 $\beta$, IFN- $\gamma$ and IL-4 in BALF were determined with sandwich ELISA kits according to the manufacturer's instruction. The total protein level in BALF was measured by the BCA method.

\section{B. RESULTS AND DISCUSSION}

1. Validation of the Method for Determining the Encapsulation Efficiency (EE) of siRNA in

\section{LPXs}

272 Free siRNA solution showed a high fluorescence intensity and linear relationship between siRNA 273 concentration and fluorescence intensity (data not shown). However, there was no free siRNA 274 detected in the LPXs suspension in the absence of HD because the lipid-bonded siRNA has lost the 275 ability to bind to the RiboGreen ${ }^{\circledR}$ fluorescence dye that exerts the fluorescence signal. By adding HD, 276 the recovery of siRNA was around $100 \%$. The results proved that in the absence of HD, the 277 RiboGreen ${ }^{\circledR}$ fluorescence dye was able to detect the free siRNA in the LPXs suspension, but not the 
siRNA associated with DOTAP, which suggested that this method was suitable to determine EE of the LPXs.

Table S1. Comparison of the recovery of siRNA in the LPXs in the presence or absence of HD $281 \quad($ mean $\pm \mathrm{SD}, \mathrm{n}=3)$.

\begin{tabular}{ll}
\hline Testing method & Recovery rate (\%) \\
\hline With HD & $101.79 \pm 2.32$ \\
Without HD & 0 \\
\hline
\end{tabular}

\section{2}

\section{Investigation of the In Vitro Release of siRNA From the LPXs and LPNs}

Figure S1 shows the in vitro long-term release of siRNA from LPNs with or without HD present in release medium. Since only free siRNA could bind to RiboGreen ${ }^{\circledR}$ RNA reagent to exert fluorescence while the bounded form of siRNA could not bind to RiboGreen ${ }^{\circledR}$ RNA reagent to exhibit detectable fluorescence, in the absence of HD solution which served as dissociation reagent, only the fraction of released siRNA in free form could be detected, and was referred to as "free siRNA". However, with the presence of HD solution, the bounded form of siRNA was set free and detected by the RiboGreen ${ }^{\circledR}$ RNA reagent. According to the results, there was negligible free siRNA during the experimental period, therefore, the amount of siRNA detected by the RiboGreen ${ }^{\circledR}$ RNA reagent with the presence of HD solution was considered equivalent to the bounded form of siRNA, and was referred to as "bound siRNA".

The large difference between the release profiles of the bound siRNA and the free siRNA suggests that the majority of siRNA was released in the form of bound siRNA instead of free siRNA (Figure S1). In addition, the released bound siRNA did not dissociate during the entire release process. The profile corresponding to bound siRNA exhibited an initial burst release followed by a sustained-release profile. The LPXs applied in the release study, however, did not show any free siRNA during the experimental process and the data was not shown. 


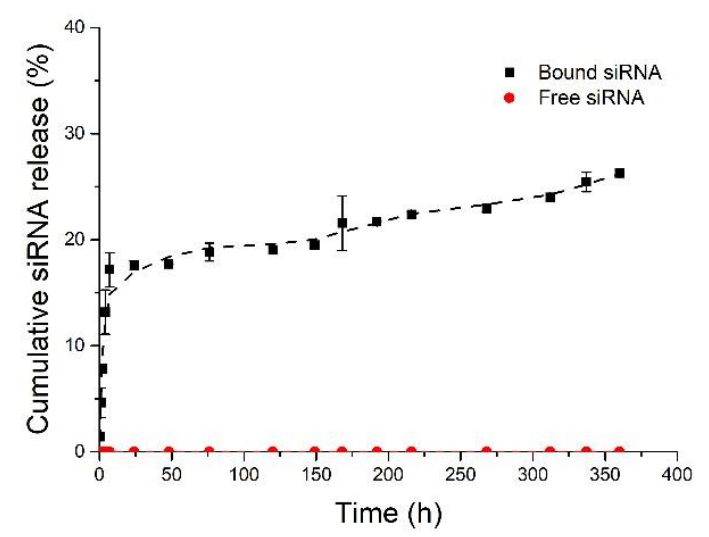

Figure S1. Cumulative release of the siRNA from LPNs.

\section{Effect of Anionic Endogenous Agent Hyaluronic Acid (HA) on Gene Silencing Performances} of LPNs and LPXs

The effect of the presence of HA in the cell media on the gene silencing effects of the two formulations was evaluated by flow cytometry (Figure S2) and Western blotting analyses (Figure S3). The results illustrated in Figure S2 showed that the gene silencing effect of LPNs (loaded with both 40 and $80 \mathrm{nM}$ siRNA) in $0.1 \%$ HA solution is similar with that in DEPC water. There are significantly reduced gene silencing efficiency of LPNs observed with the presence of $0.3 \%$ and $0.5 \%$ HA solution as compared to that in DEPC water though. In contrast, a significant reduction of gene silencing efficiency of LPXs can already be observed with the presence of $0.1 \%$ HA solutions as compared to that in DEPC water. The difference in the negative impact of HA on the two kinds of nanoparticle formulations seemed to be more obvious when comparing the EGFP fluorescence percentages of the two kinds of nanoparticle formulations at the siRNA concentration of $80 \mathrm{nM}$. As shown in Figure S2, the EGFP fluorescence percentage of LPNs was increased from ca. 50\% in DEPC water to $70 \%$ in $0.5 \%$ HA solution. In contrast, the EGFP fluorescence percentage of LPXs was increased from ca. $40 \%$ in DEPC water to ca. $80 \%$ in $0.5 \%$ HA solution. It implies that the negative impact of HA on the gene silencing performance of LPXs is more profound than the negative impact of HA on LPNs. In addition, the images of Western blot were processed with gray 
318 level analysis using the Image $\mathrm{J}$ software (https://imagej.nih.gov/ij/, NIH, WA, USA), the results 319 were shown in Figure S3E. Both the cellular update efficiency and the gene silencing efficiency of 320 the two kinds of nanoparticles were impaired with an increase in the concentration of HA. In 321 addition, the negative impact of HA on the performance of LPXs seems to be more profound than on 322 the performance of LPNs, especially at the high siRNA concentration, i.e. 80nM.

323 Considering the complexed microenvironment in the lung, where various endogenous materials especially anionic agents, such as dipalmitoylphosphatidylcholine, phosphatidylglycerol, and HA may interact with the positively charged LPXs and LPNs, resulting in aggregation of the nanoparticles and/or dissociation of LPXs, a simplified study was carried out by incubating LPXs and LPNs with HA, respectively to study the effects of HA on the gene silencing performances of the two formulations. HA was chosen in this preliminary study because of its negative to neutral charge, mimicking the negative to neutral in vivo microenvironment. It has also been reported that the HA level in the lung increases in some disease conditions such as asthma, COPD, edema and fibrosis, ${ }^{3}$ suggesting that HA may be an important endogenous substance influencing the effect of inhaled formulations. The preliminary study showed that the gene silencing effects of both LPXs and LPNs were compromised in the presence of HA. Nevertheless, the negative impact of HA on LPXs was more profound than on the LPNs. This suggests that PLGA could protect LPXs from dissociation in the presence of HA. In summary, LPNs exhibited less efficient gene silencing, less cytotoxic, and are more stable than LPXs when challenged by anionic naturally existing molecules in the in vitro study. 


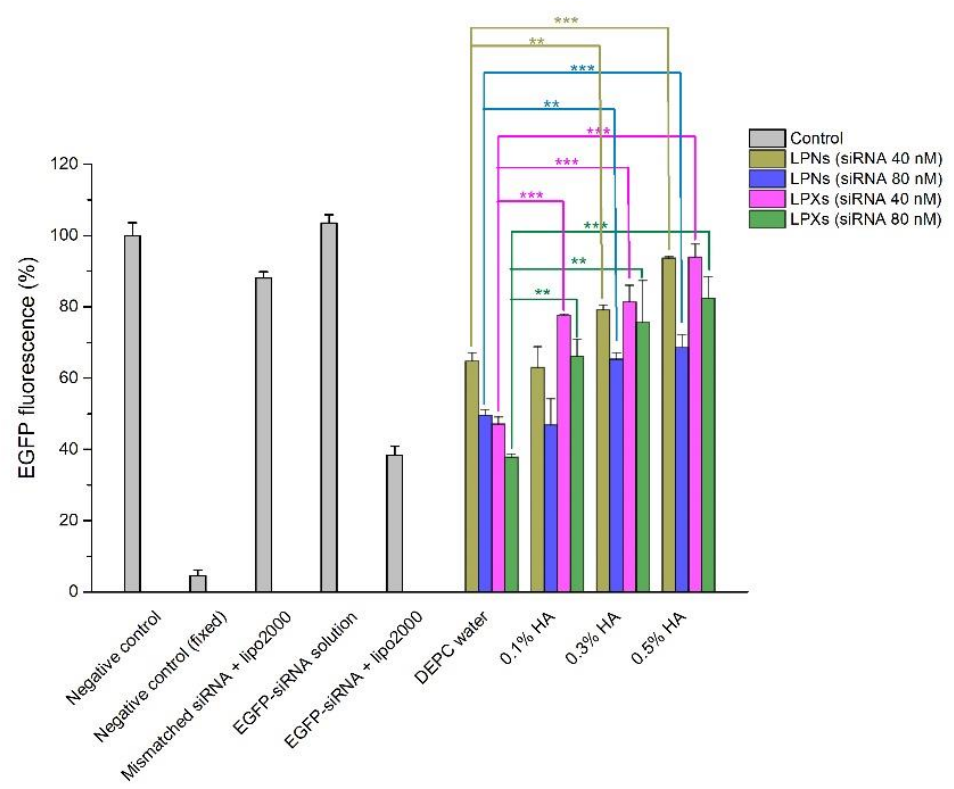

338 Figure S2. In vitro gene silencing efficiency of the LPXs and LPNs in the presence of 0 to $0.5 \%$ HA 339 measured by flow cytometry $(n=3)$. Statistic difference studies between nanoparticles with the same 340 concentration of siRNA and treated with different amounts of HA were conducted relative to DEPC 341 water treated groups of each concentration of siRNA respectively, *P $\leqslant 0.05, * * \mathrm{P} \leqslant 0.01, * * * \mathrm{P} \leqslant$ 3420.001.

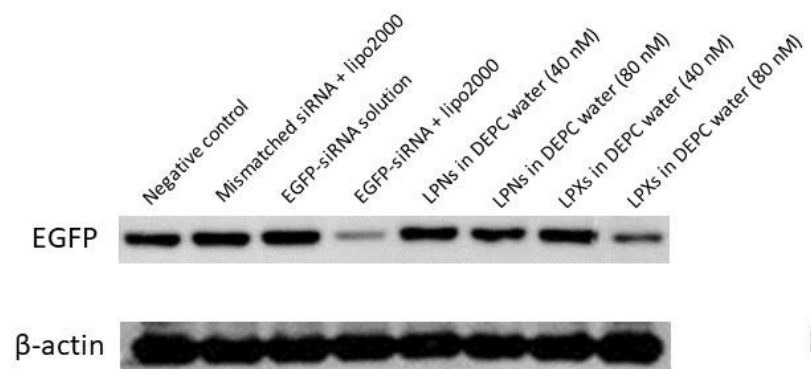

(A)

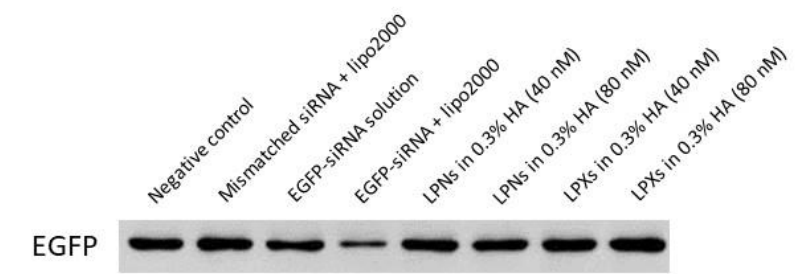

$\beta$-actin

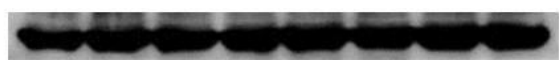

(C)

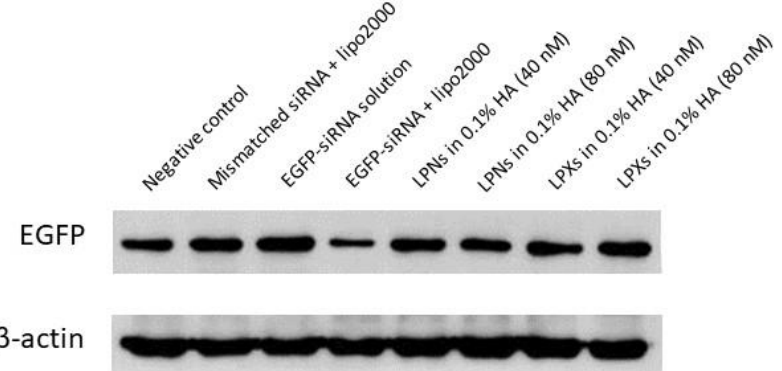

(B)

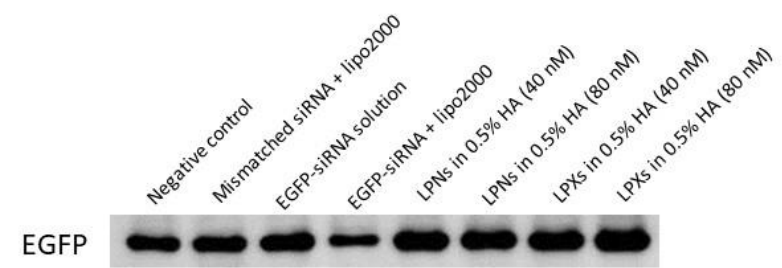

$\beta$-actin

(D) 


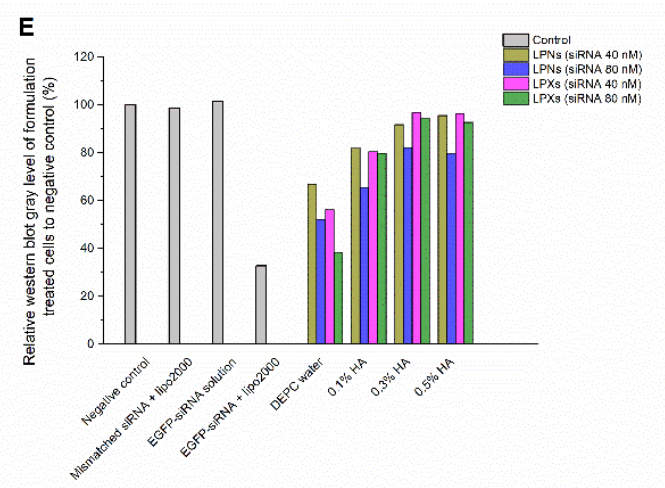

346 Figure S3. In vitro gene silencing efficiency of the LPXs and LPNs in the presence of (A) 0\%, (B) $3470.1 \%$, ( C) $0.3 \%$ and (D) $0.5 \%$ HA measured by Western blotting. The percentage of the gray level of 348 Western blot images of EGFP protein extracted from cells treated with LPXs and LPNs in the 349 presence of $0 \%, 0.1 \%, 0.3 \%$ and $0.5 \%$ HA relative to blank medium treated negative control (E).

\section{Colloidal Stability Test of LPNs and LPXs}

351 As shown in Figure S4, the sizes of both LPNs and LPXs were increased with an increase in the 352 incubation time in SLF. However, the increase in the size of LPXs is more profound than that of 353 LPNs, indicating poor colloidal stability of LPXs as compared to LPNs. After $1 \mathrm{~h}$ and $24 \mathrm{~h}$ of 354 incubation in SLF, the size of LPXs were increased ca. 2.2 and 8.9 folds, respectively. In contrast, the 355 increase in the size of LPNs after $1 \mathrm{~h}$ and $24 \mathrm{~h}$ of incubation in SLF were ca. 1.4 and 2.1 folds, 356 respectively. Meanwhile, the zeta potential of both LPNs and LPXs were decreased after incubating 357 in SLF.
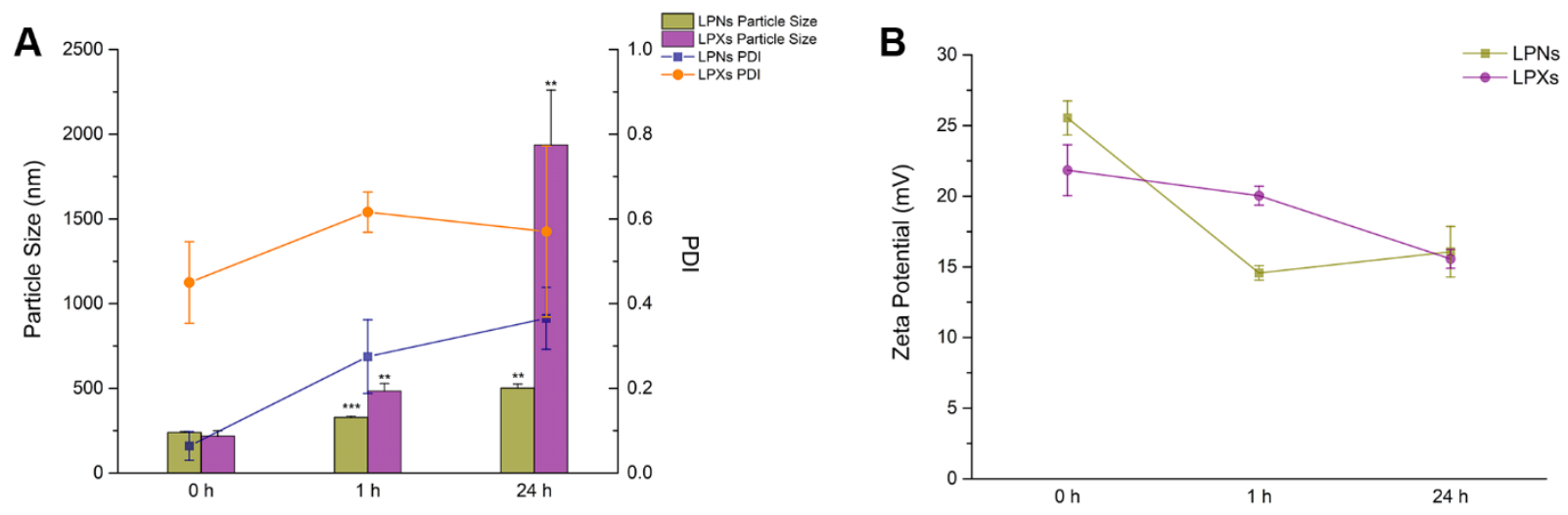
359 Figure S4. Particle size and PDI of LPNs and LPXs after incubating in SLF (A). Zeta potential of 360 LPNs and LPXs after incubating in SLF (B). Statistical analyses of particle sizes of LPNs and LPXs 361 at $1 \mathrm{~h}$ and $24 \mathrm{~h}$ were conducted relative to the particle sizes of LPNs and LPXs at $0 \mathrm{~h}$, respectively. $362 * \mathrm{P} \leqslant 0.05, * * \mathrm{P} \leqslant 0.01, * * * \mathrm{P} \leqslant 0.001$

A

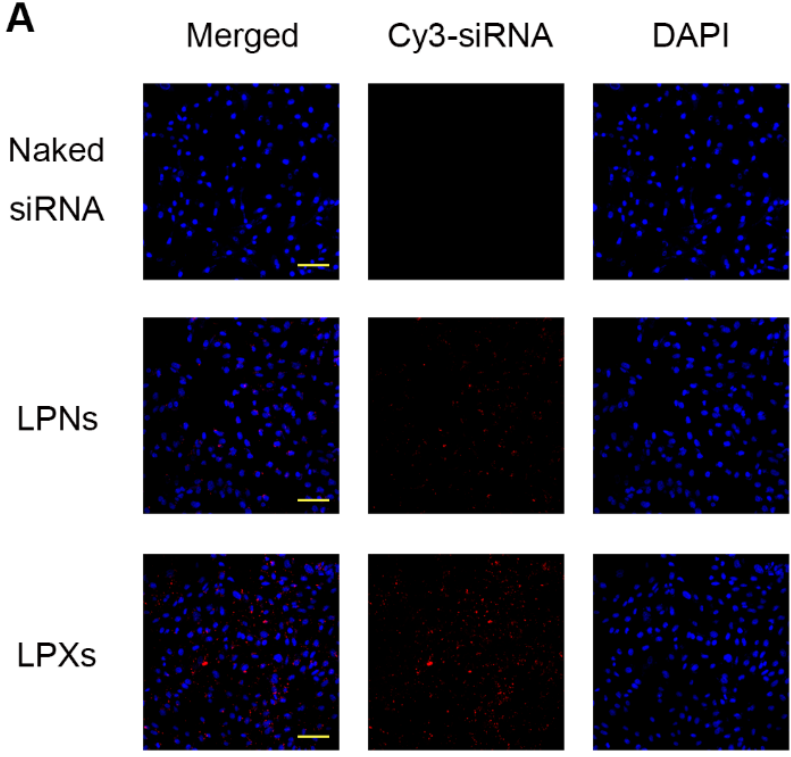

B

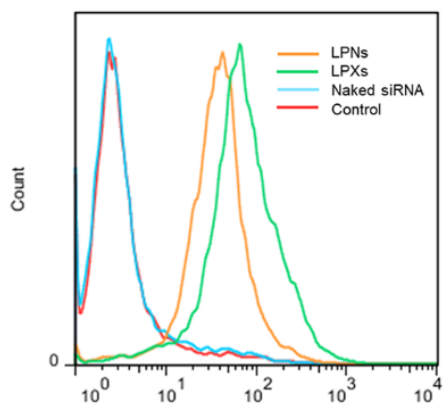

суз
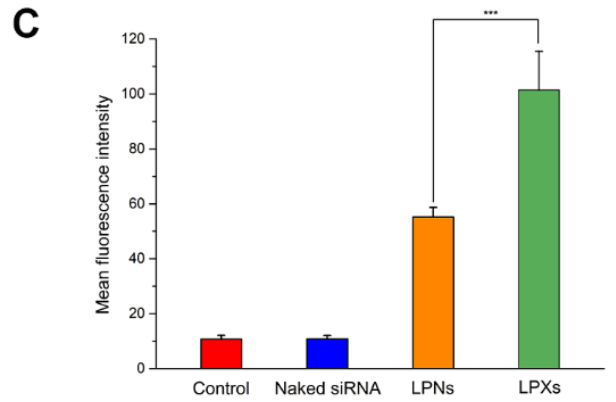

Figure S5. CLSM images of the A549-EGFP cells incubated with Cy3-siRNA loaded LPNs or LPXs (A), scale bar $=50 \mu \mathrm{m}$; Representative flow cytometry peaks of different groups (B); Flow cytometry results of the cellular uptake of different formulations (C). Statistical difference studies of 
374 MFI were conducted between LPNs and LPXs. *P $\leqslant 0.05, * * \mathrm{P} \leqslant 0.01, * * * \mathrm{P} \leqslant 0.001$.

\section{6. Genotyping of EGFP Transgenic Mice}

376 According to the images of agarose gel (Figure S6), it could be concluded that all of the tested mice 377 were gene positive and have comparative EGFP gene expression levels. The mice were then divided 378 into five groups randomly to avoid the influence of individual differences.
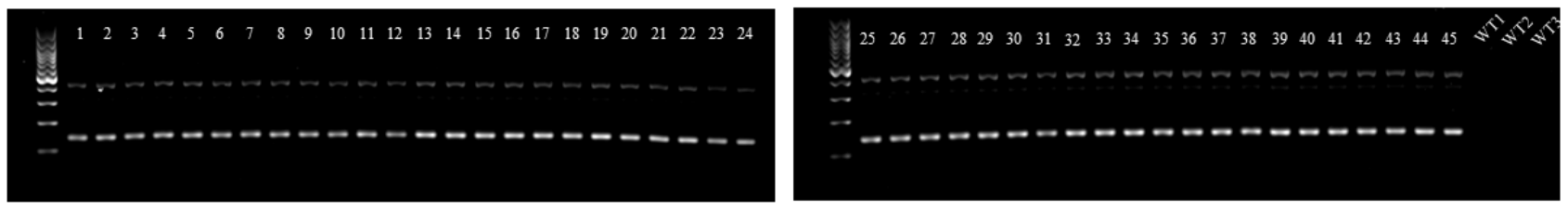

380 Figure S6. Agarose gel electrophoresis showing the PCR genotyping for mice.

381 7. Comparation of Transgenic Mice Tissue Fluorescence and Wild Type Mice Tissue 382 Autofluorescence

383 According to the IVIS images (Figure S7), the green fluorescence signal of the EGFP transgenic 384 mouse tissue and tissue lysate were two to three orders of magnitude higher than that of the WT 385 mouse. Therefore, the autofluorescence is not likely to interrupt with the fluorescence intensity 386 results in our case. 
A

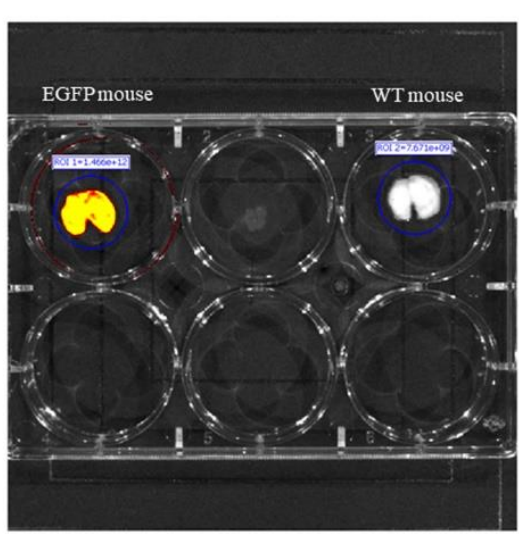

B

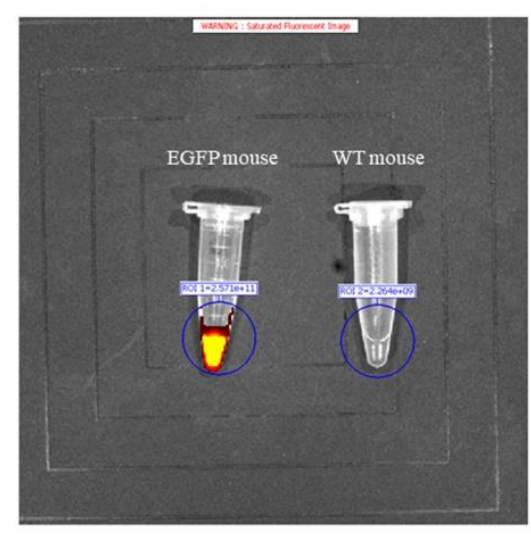

C

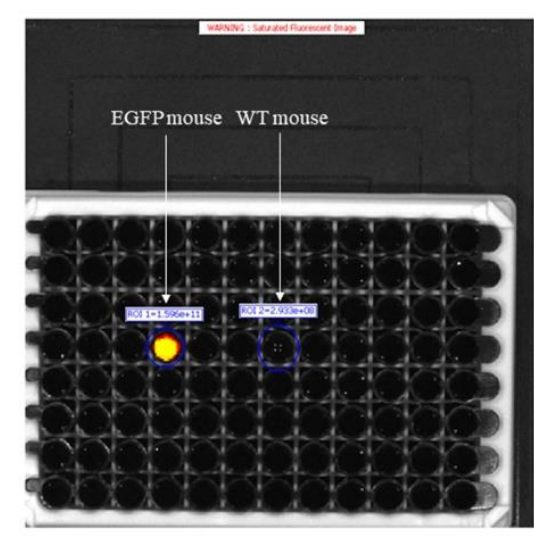

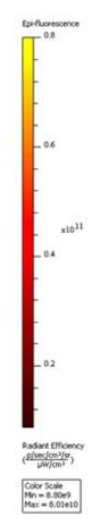
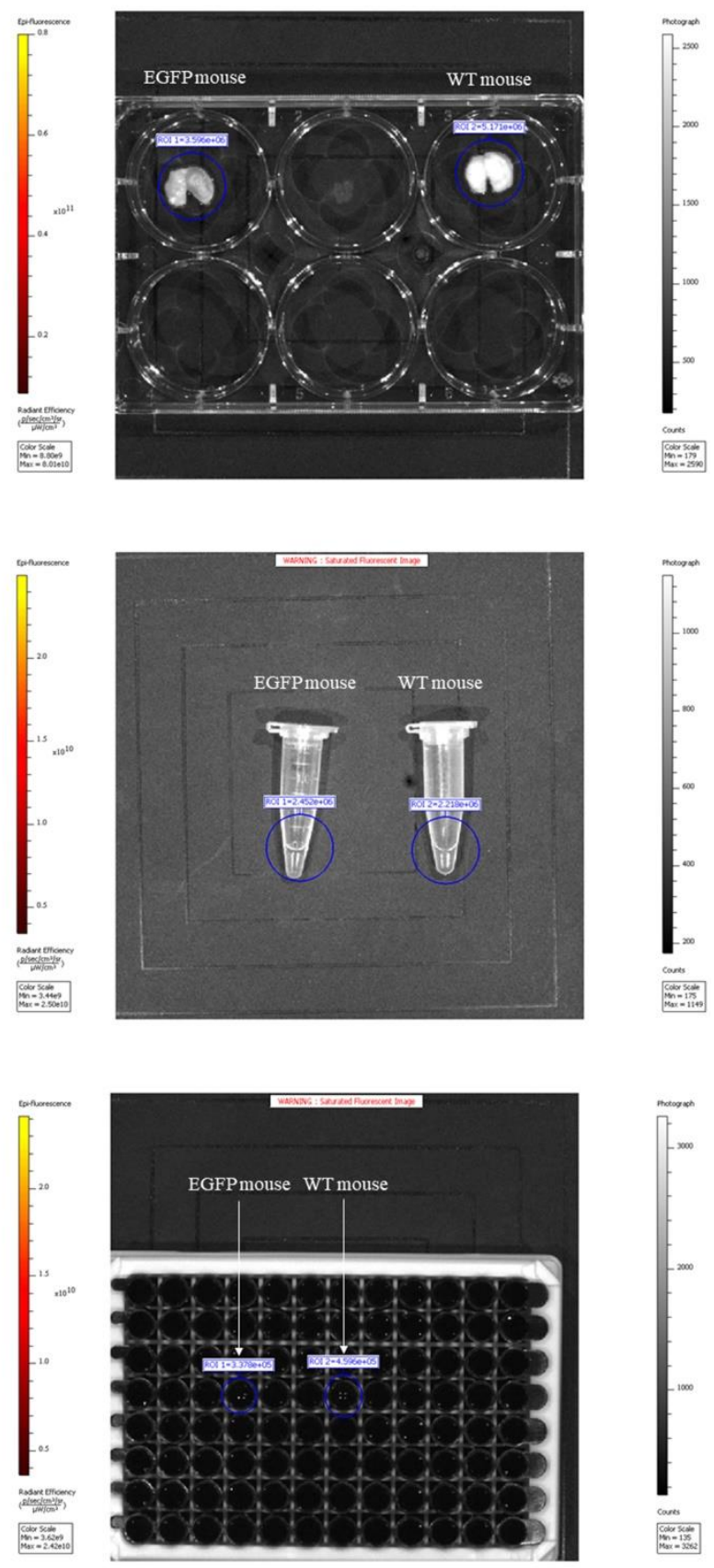

388 Figure S7. IVIS images of lung tissues (A); IVIS images of lung tissue lysate in Eppendorf tubes (B);

389 IVIS images of lung tissue lysate in 96-well plates (C).

8. Verification of the Linear Correlation between Total Protein Concentration and

\section{Fluorescence Intensity of EGFP in Lung Tissues}

392 Figure S8 shows a linear correlation relationship between the total protein concentration and the 
393 fluorescence intensities of 4 representative tissue samples with the regression coefficients $\mathrm{R}^{2}$ in the 394 range of 0.9738 to 0.9903.
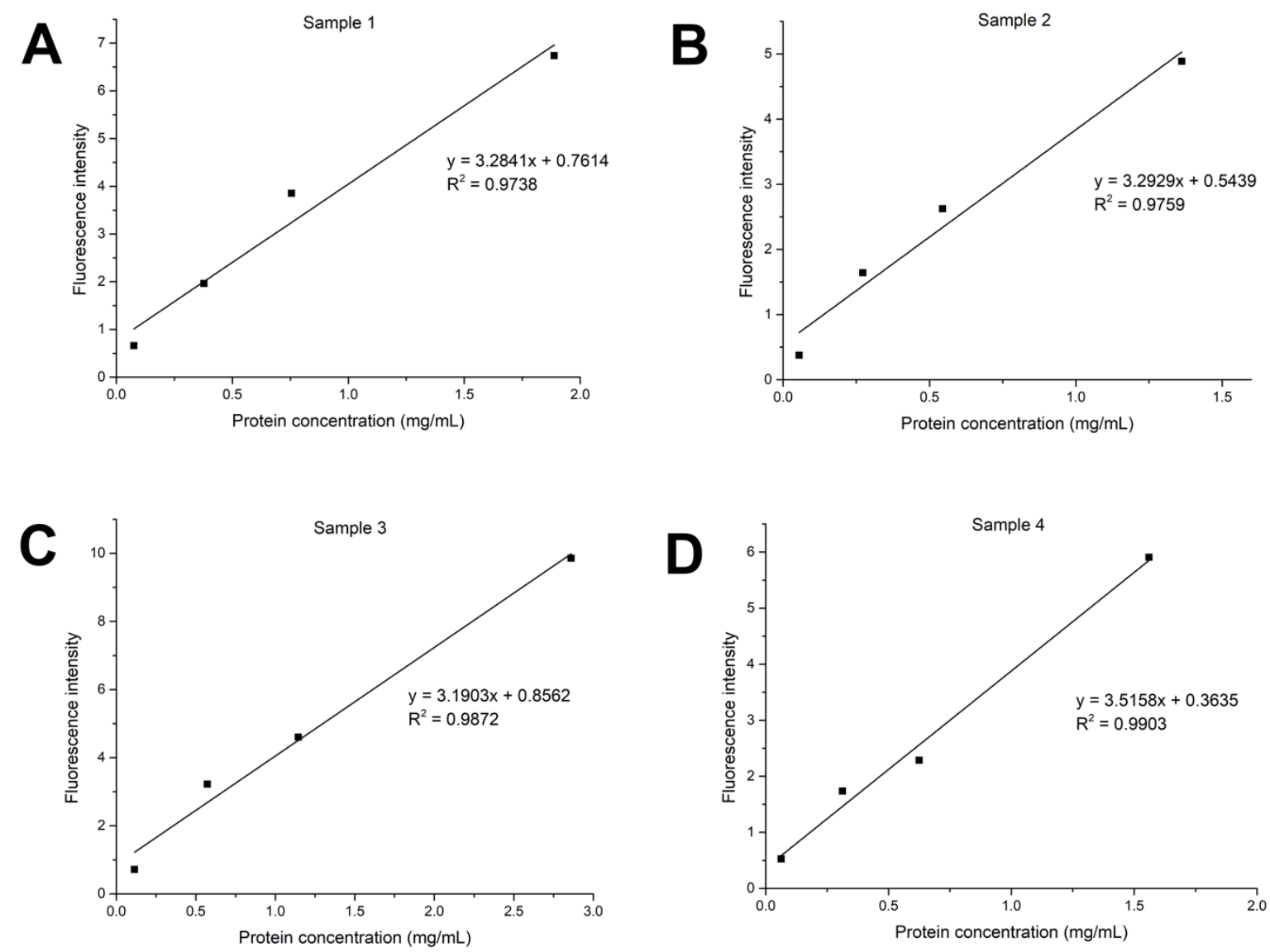

397 Figure S8. Linear fitting of protein concentration and fluorescence intensity of the lung tissue lysate.

399 The dot plot and fluorescence intensities of different channels from representative samples are shown in Figure S9. The gating strategies were kept constant for all measurements. 


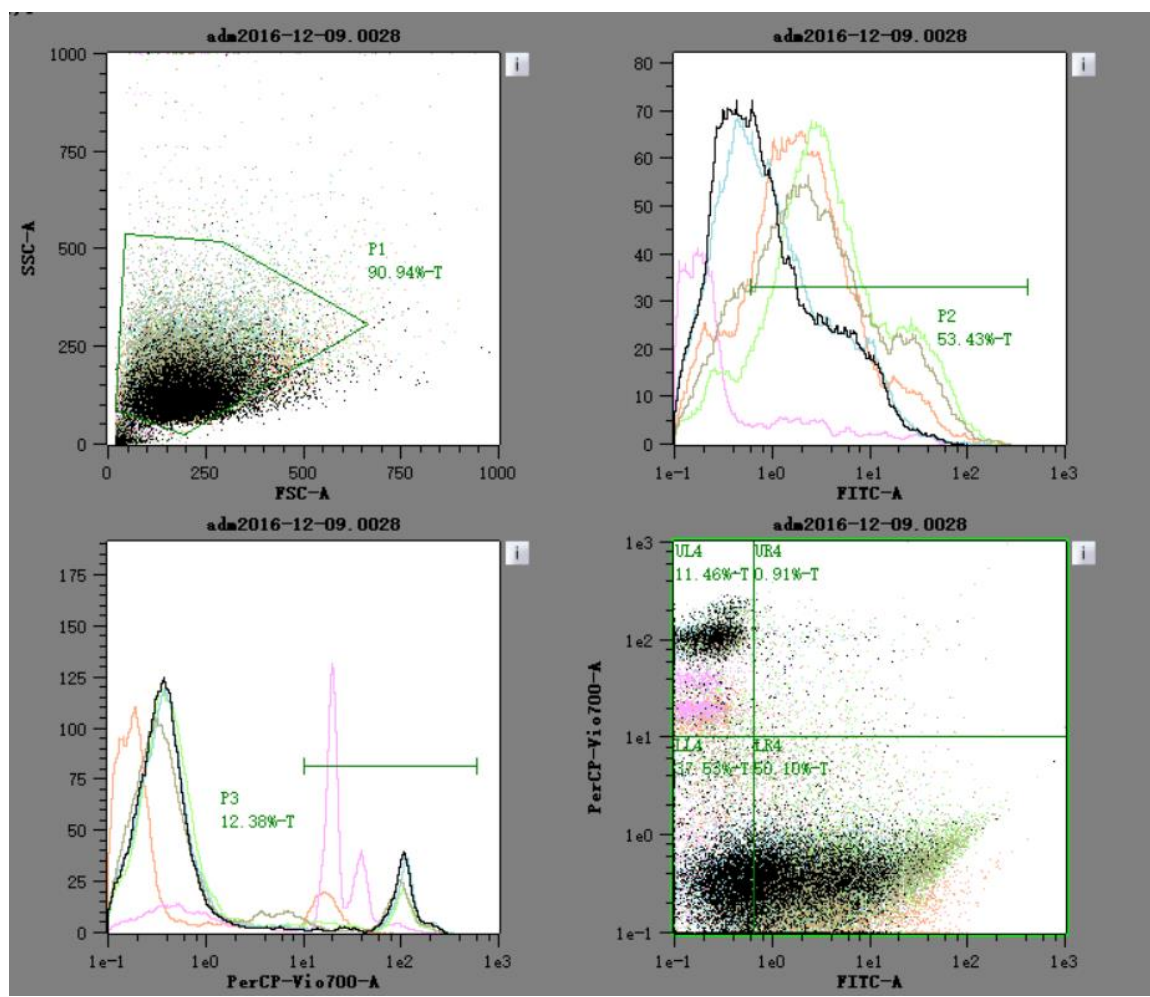

402 Figure S9. Dot plots and fluorescence intensity peaks of representative samples. Orange: negative 403 control; pink: negative control (fixed); green: mismatched siRNA; blue: EGFP siRNA transfection; 404 brown: EGFP siRNA solution; black: representative sample.

\section{10. Western Blotting and CLSM Images of Lung Tissue}

406 The results of Western blotting and CLSM images for different lung lobes are presented in Figure 407 S10 and Figure S11, respectively. The procedures of Western blotting and CLSM imaging are 408 described in section 2.6, and the relevant results and discussion are provided in section 3.2. 
A

Day 1
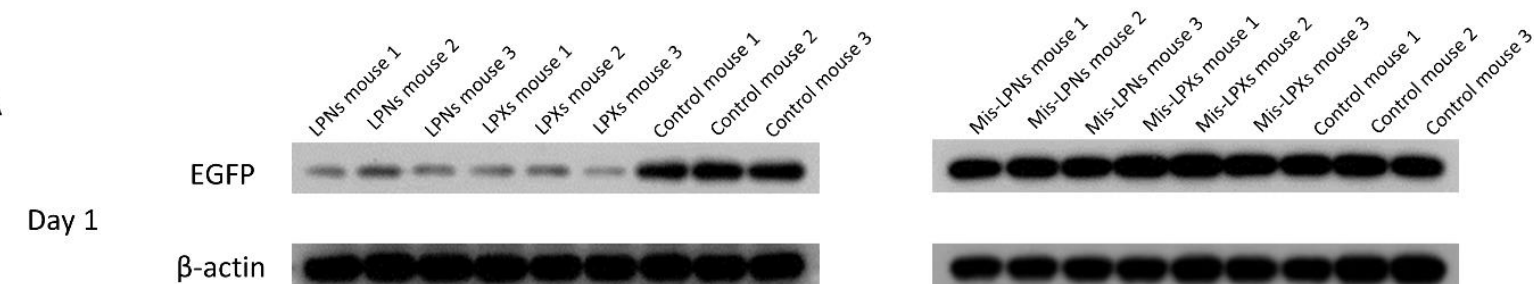

$\beta$-actin
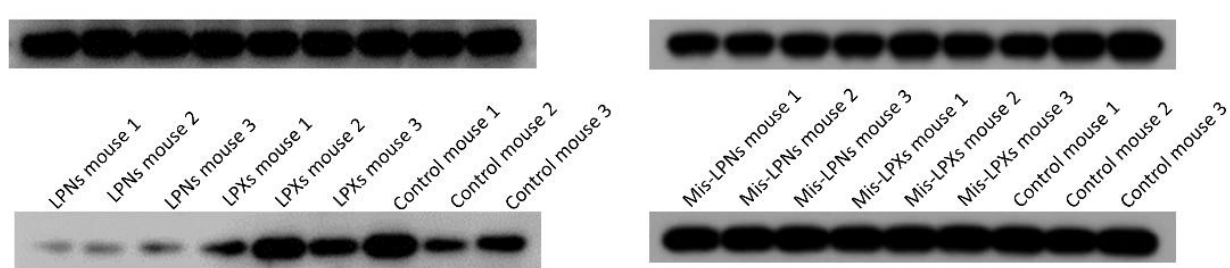

Day 6

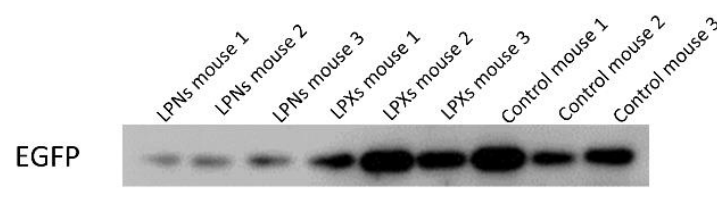

$\beta$-actin
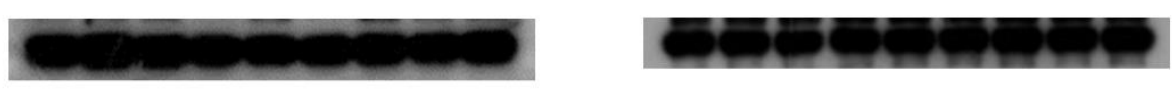

$$
\beta \text {-actin }
$$

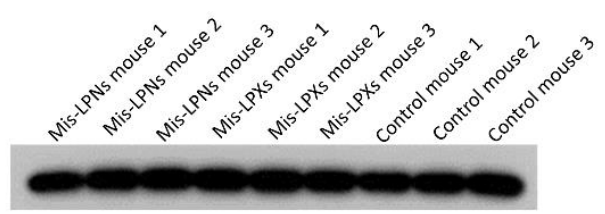

B

Day 1

EGFP
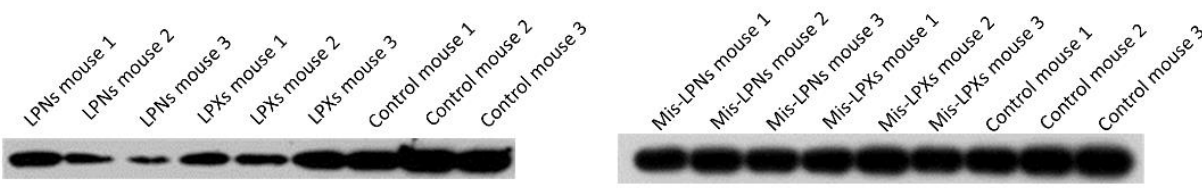

$\beta$-actin
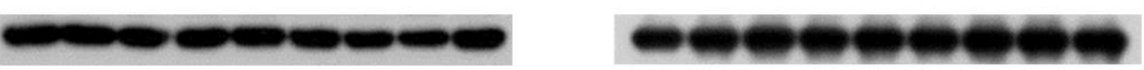

$\beta$-actin

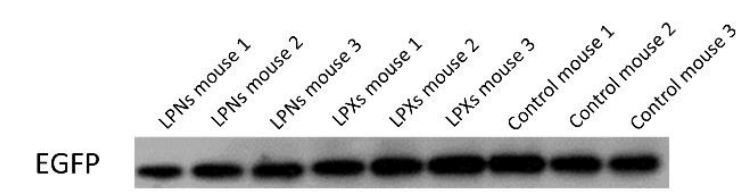

Day 6

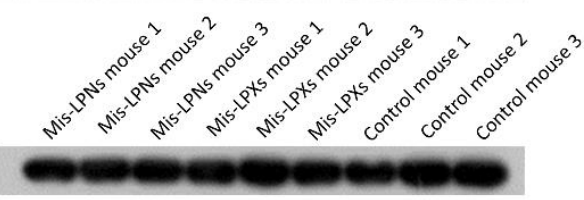

Day 11

$\beta$-actin
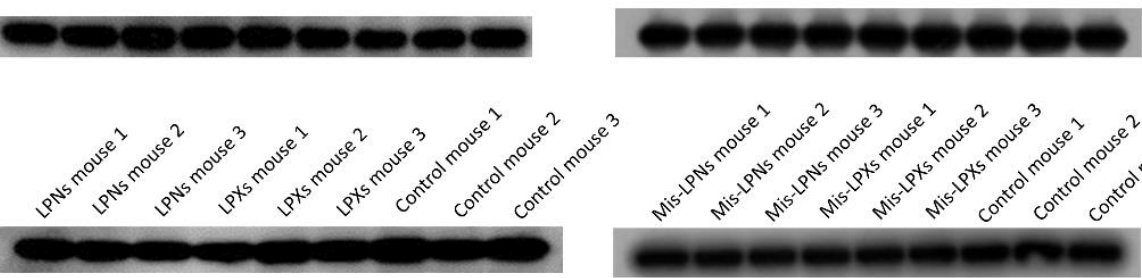

$$
\text { EGFP }
$$

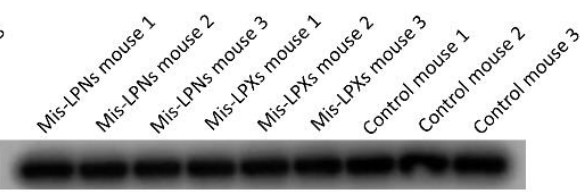


C

Day 1

Day 6
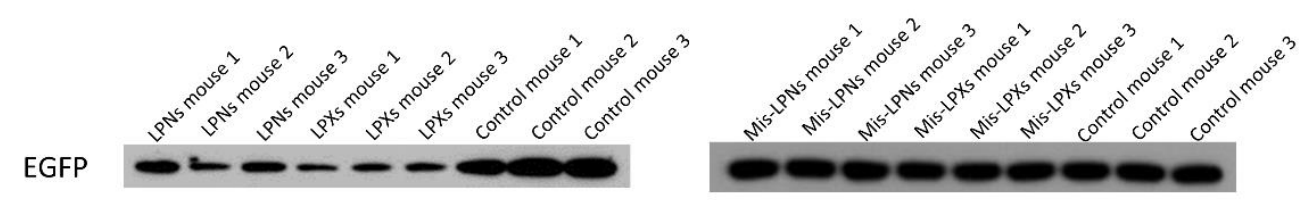

$\beta$-actin
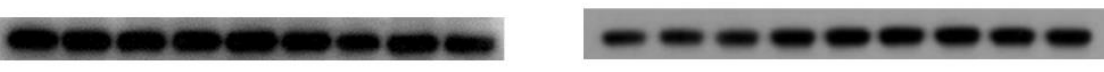

$\beta$-actin
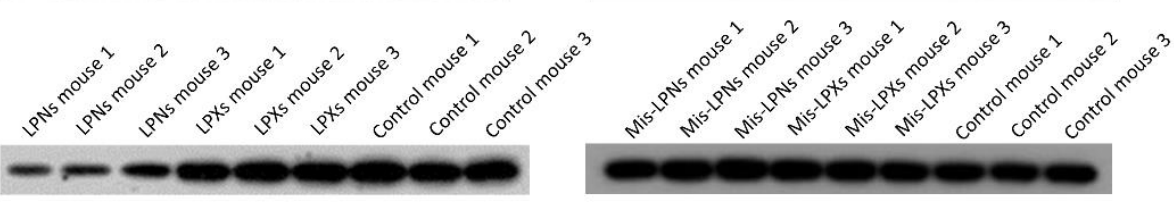

$\beta$-actin

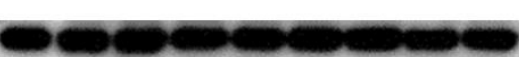

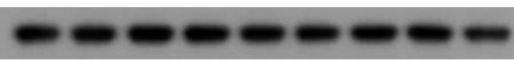

411

Day 11
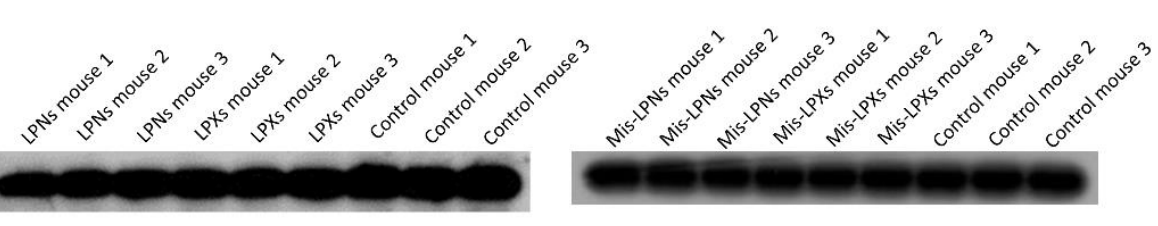

$\beta$-actin
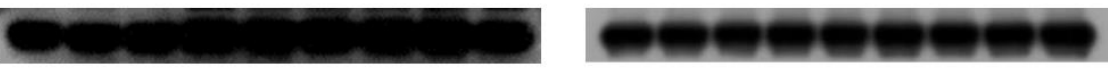

D

Day 1

$\beta$-actin
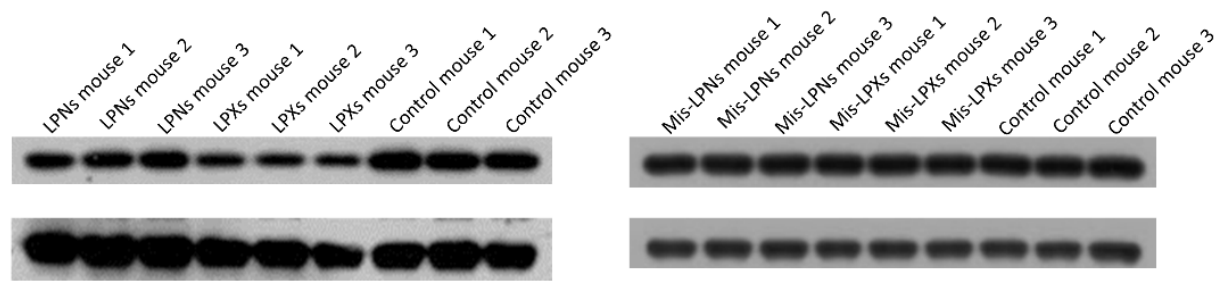

Day 6
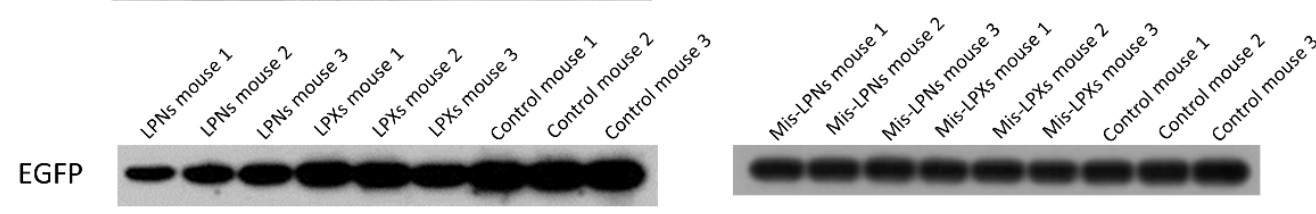

$\beta$-actin

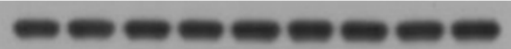

Day 11
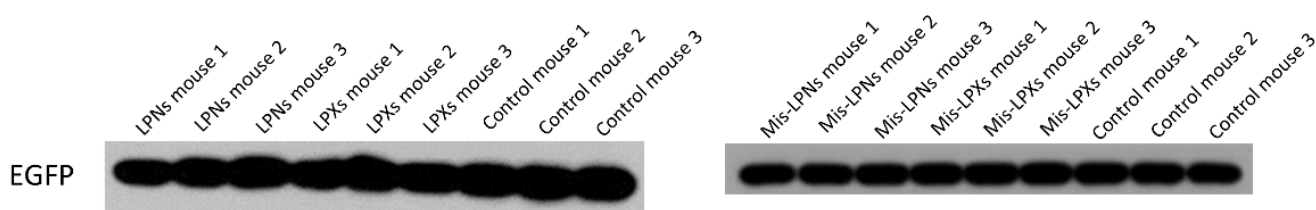

$\beta$-actin

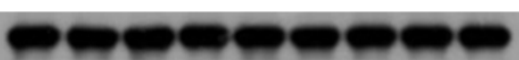




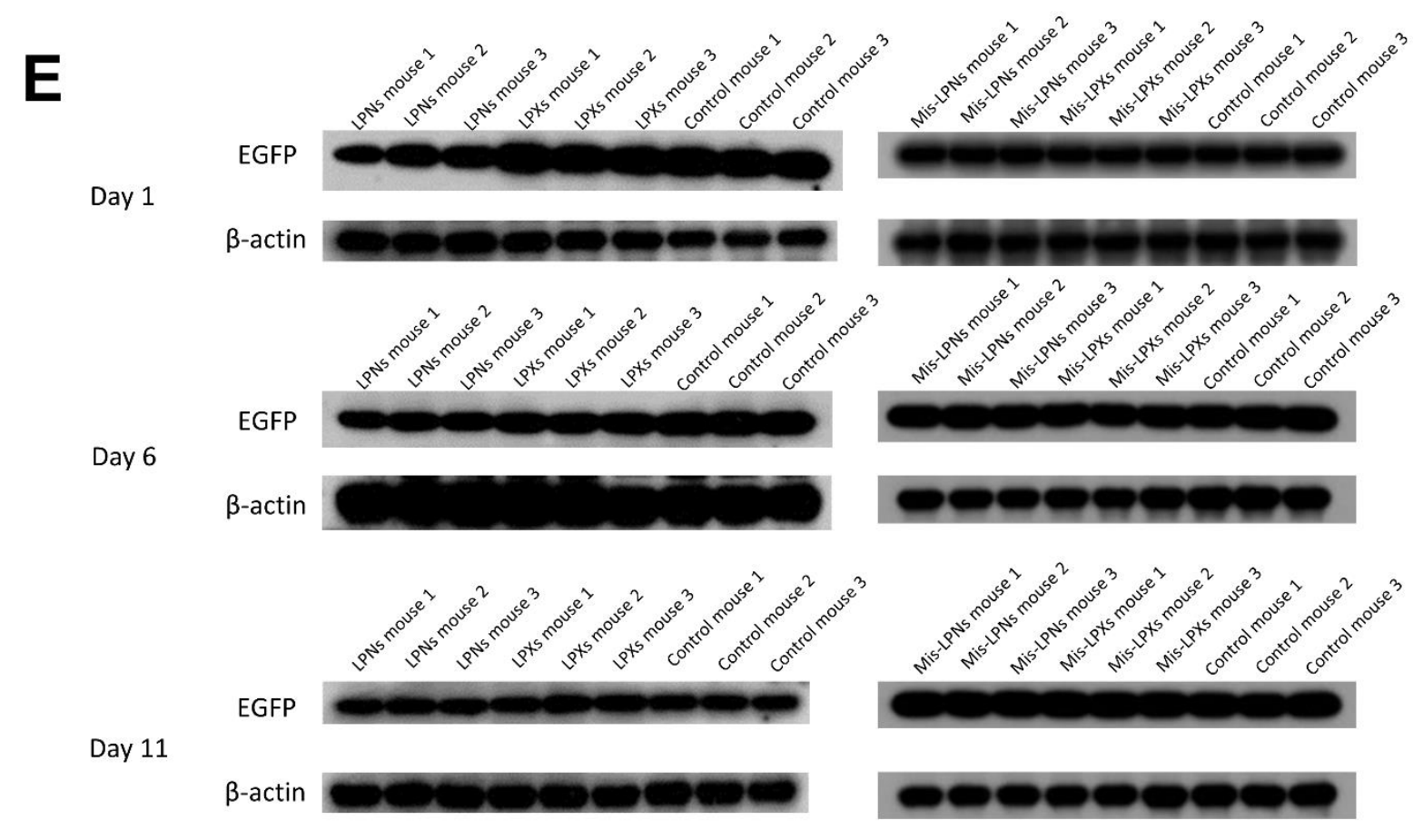

414 Figure S10. Western blotting of protein in the supernatants of the lung tissue lysates. (A) left lung, 415 (B) superior lobe of right lung, (C) middle lobe of right lung, (D) inferior lobe of right lung and (E) 416 post-caval lobe.

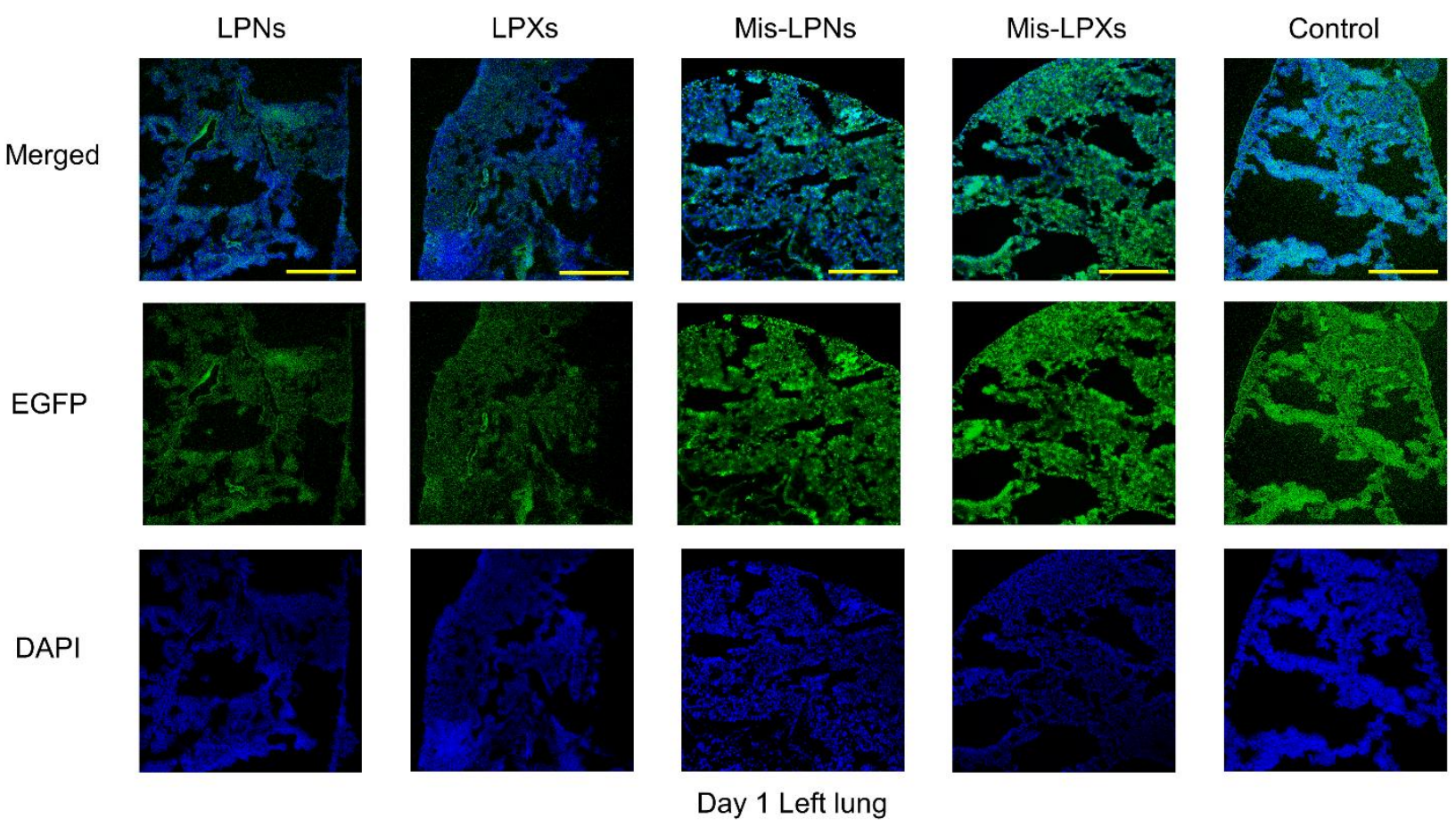




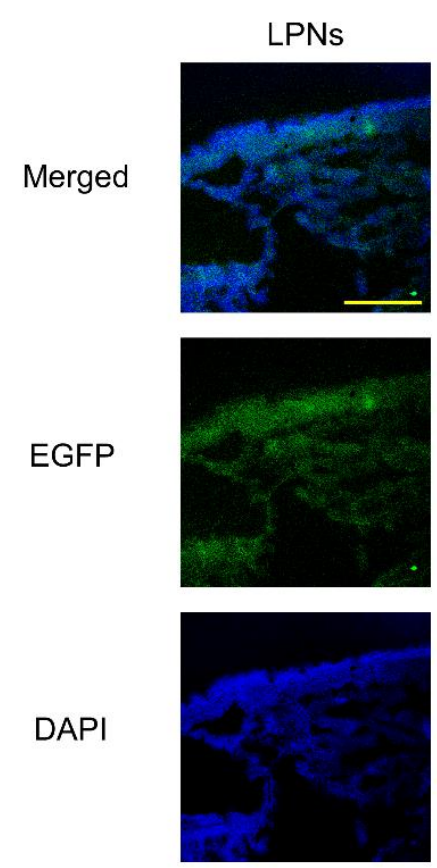

418
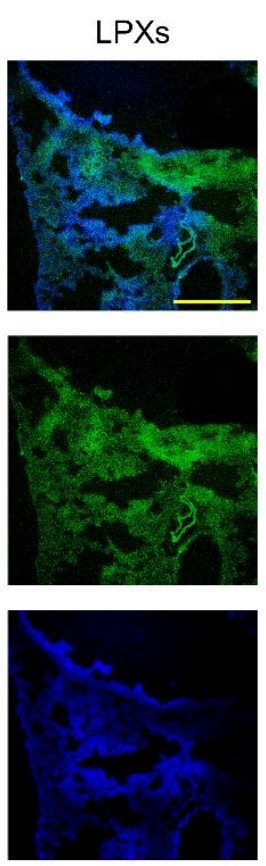

Day 1 Superior lobe of right lung
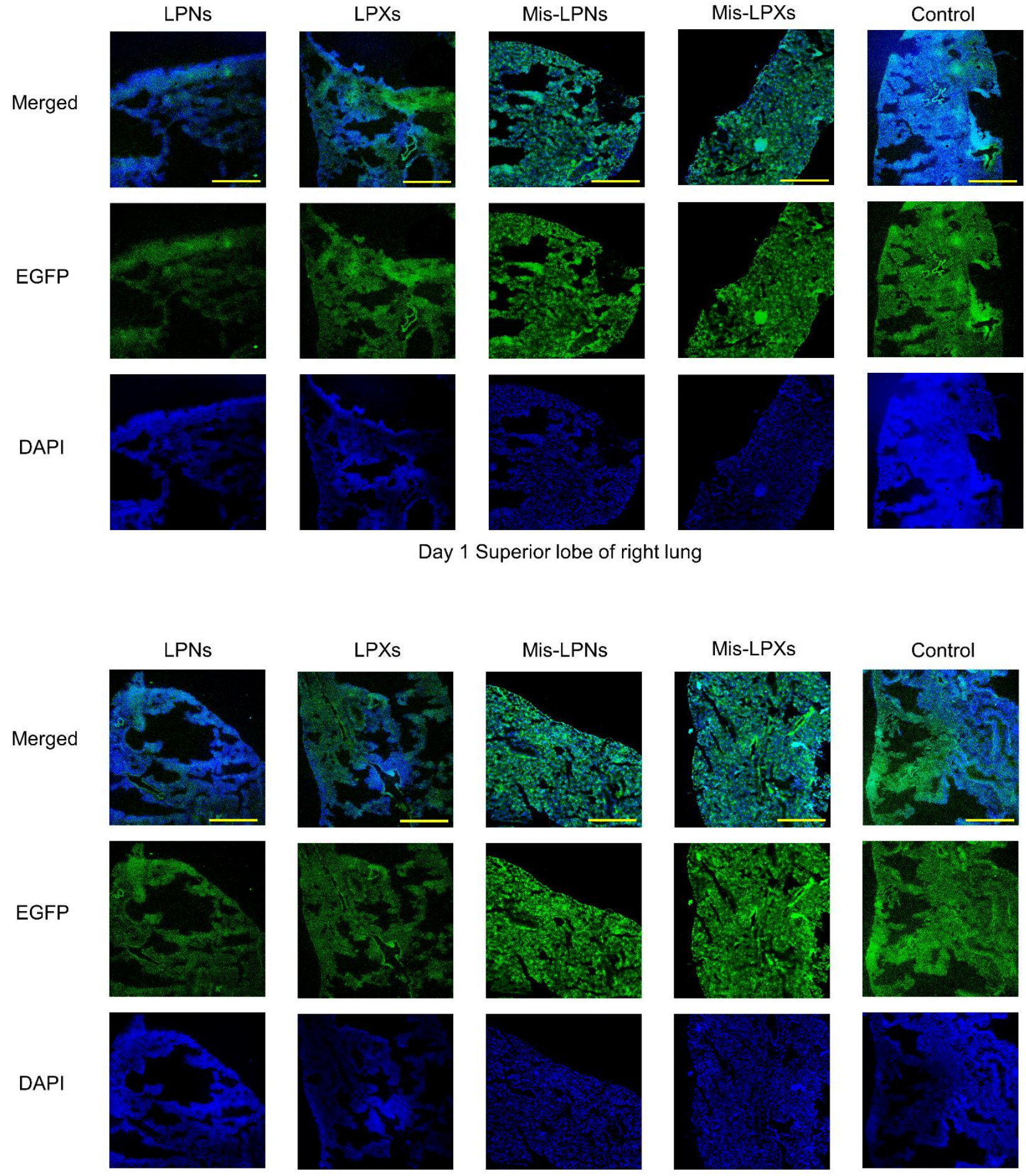
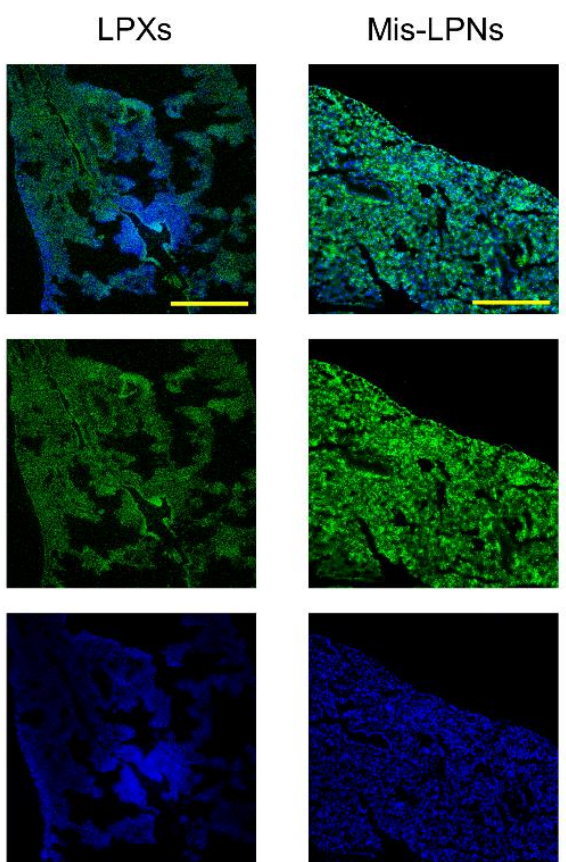

Day 1 Middle lobe of right lung
Mis-LPXs
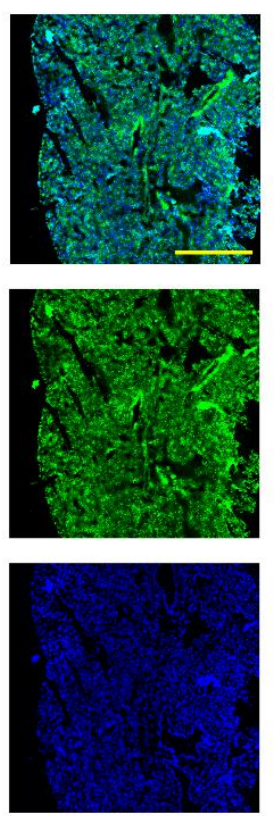

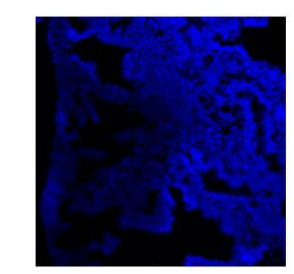

Control
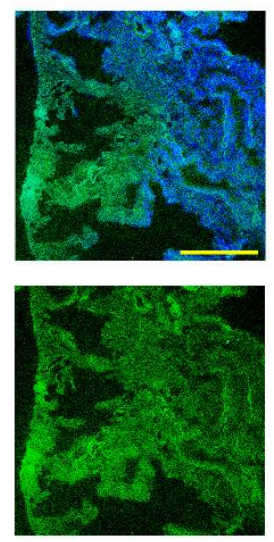

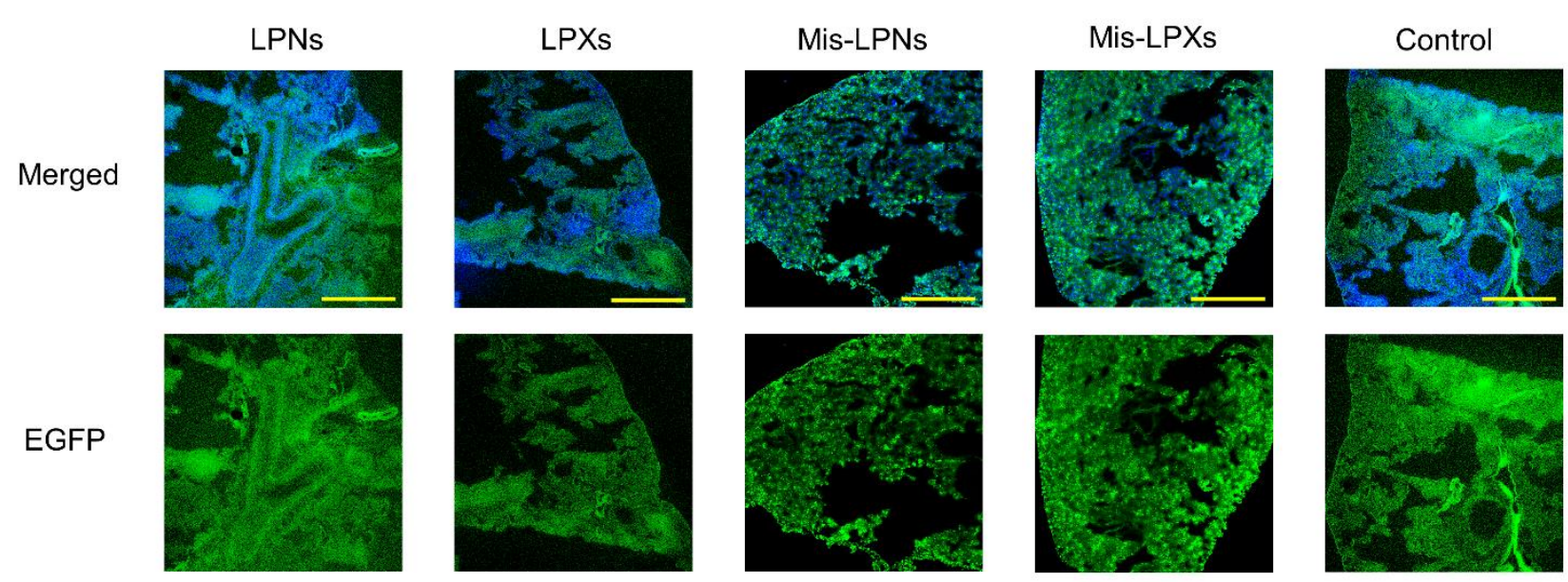

DAPI
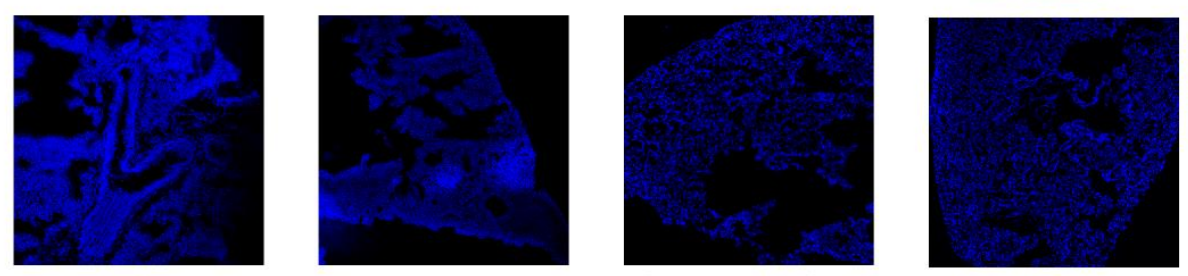

Day 1 Inferior lobe of right lung
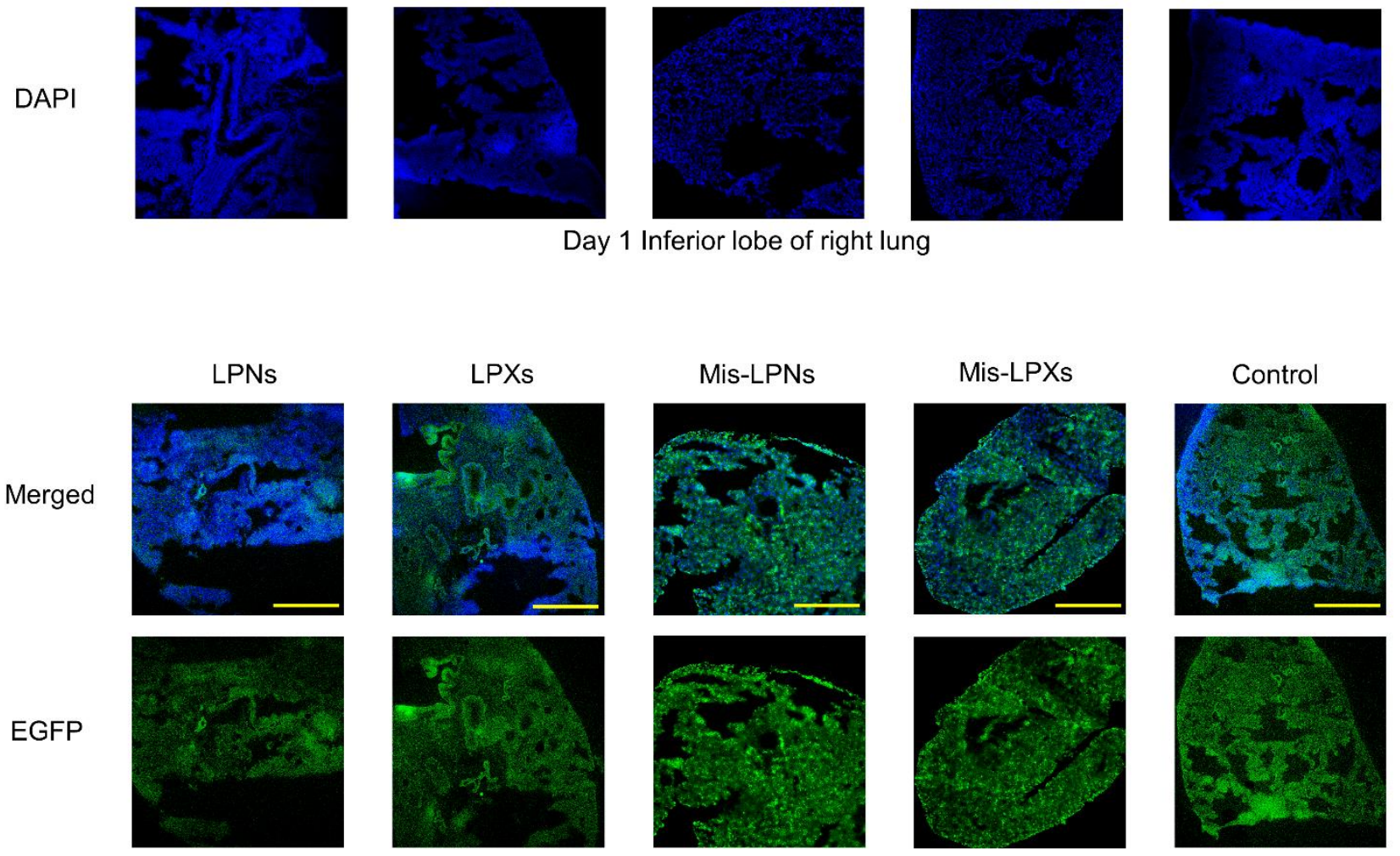

DAPI
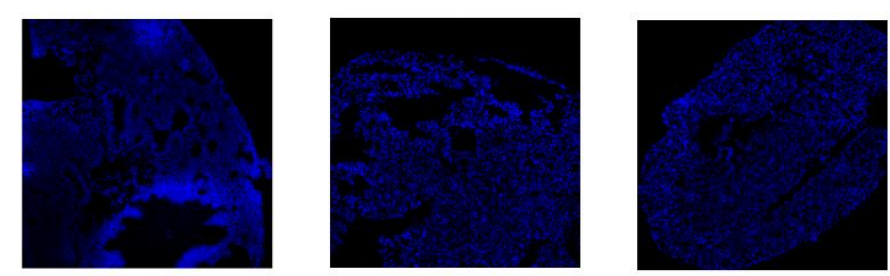

Day 1 Post-caval lobe

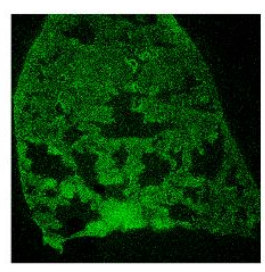



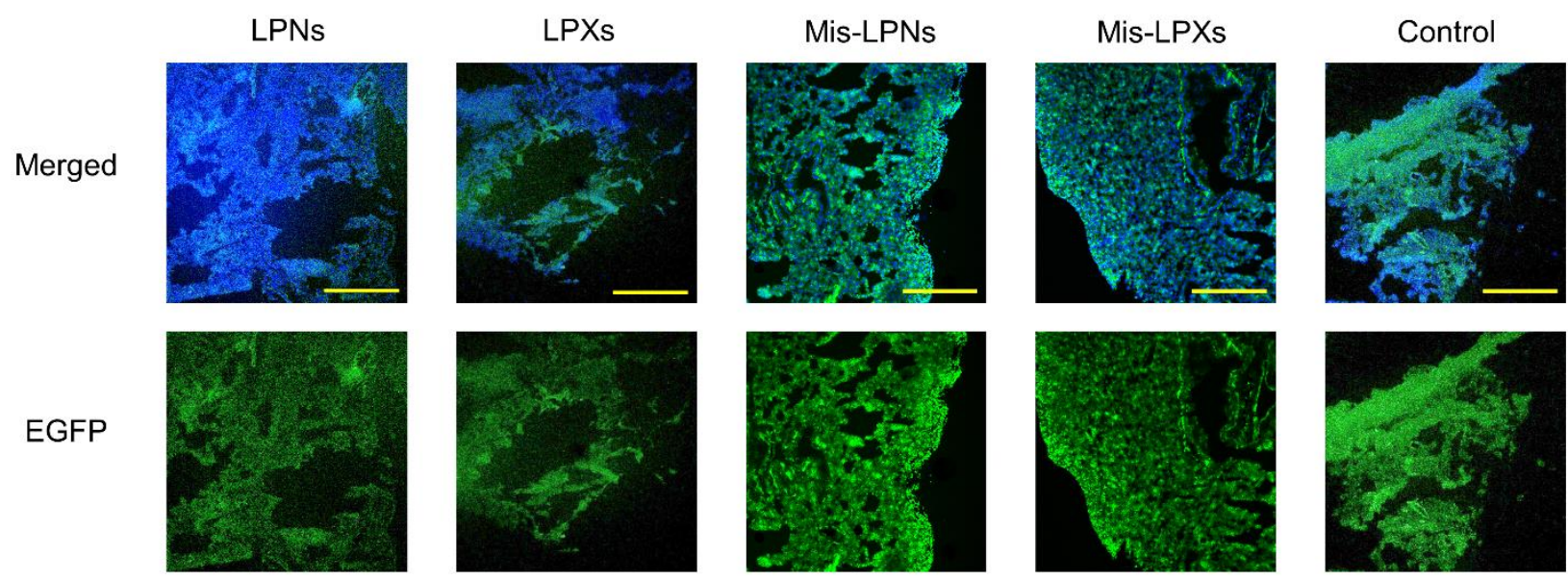

DAPI
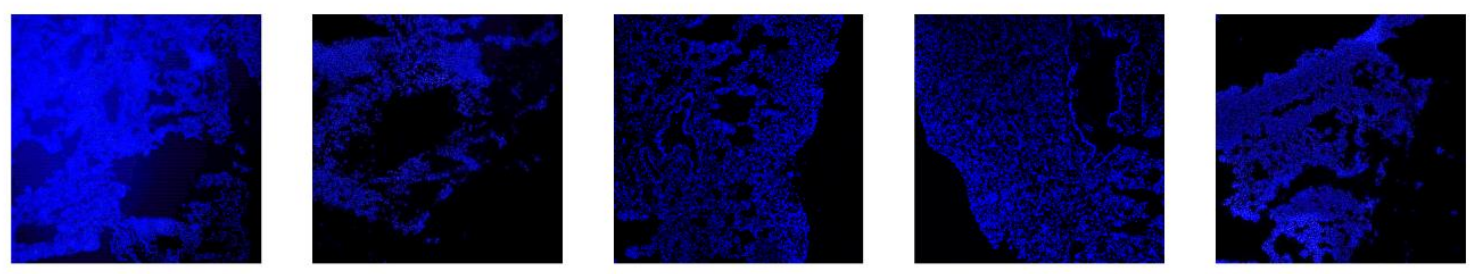

Day 6 Left lung
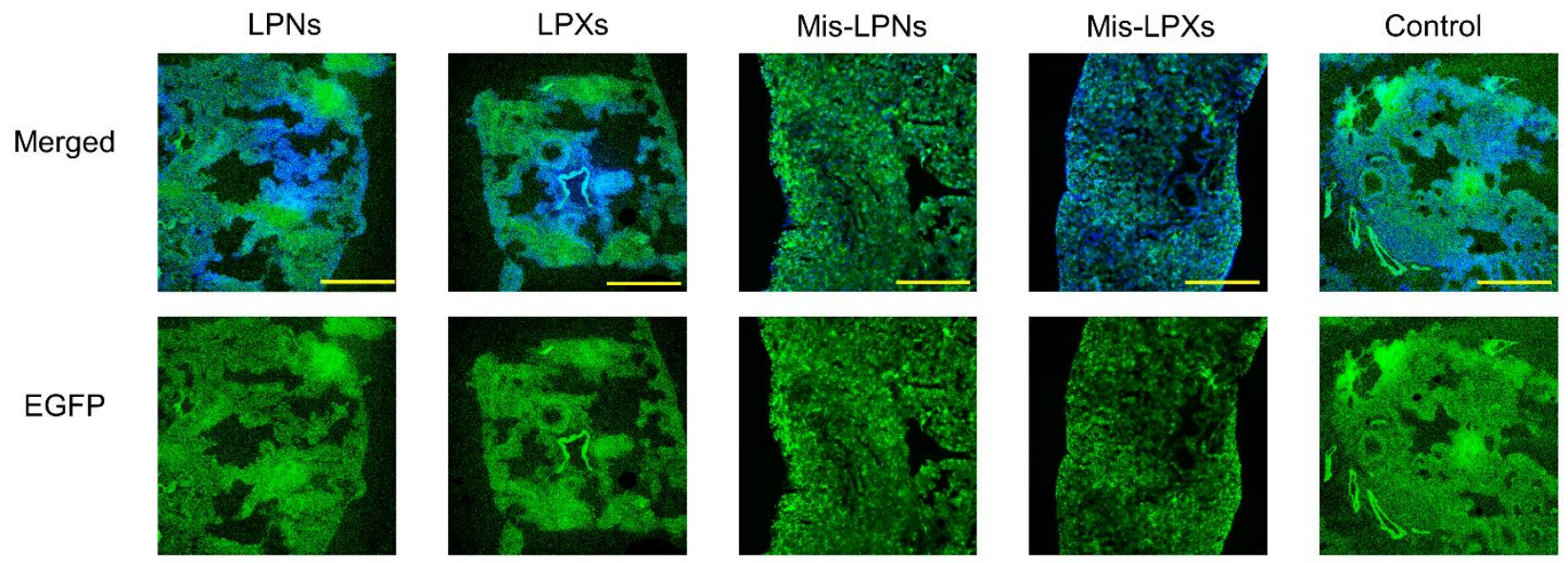

DAPI
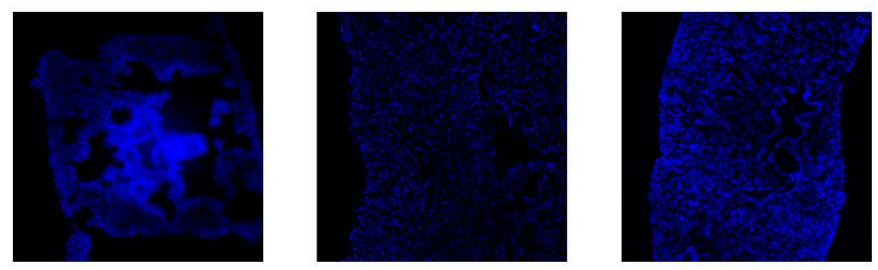

Day 6 Superior lobe of right lung

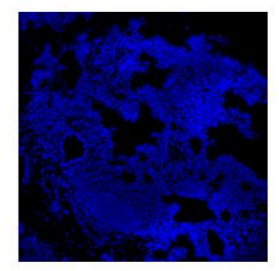



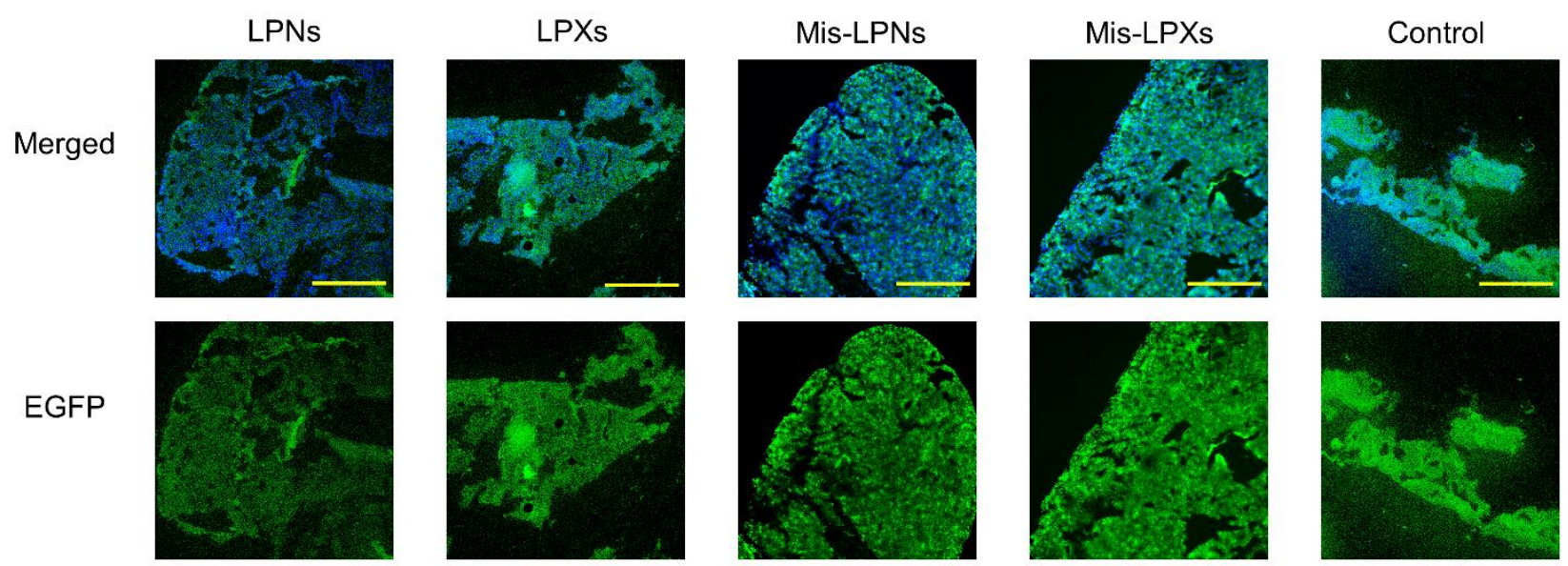

DAPI
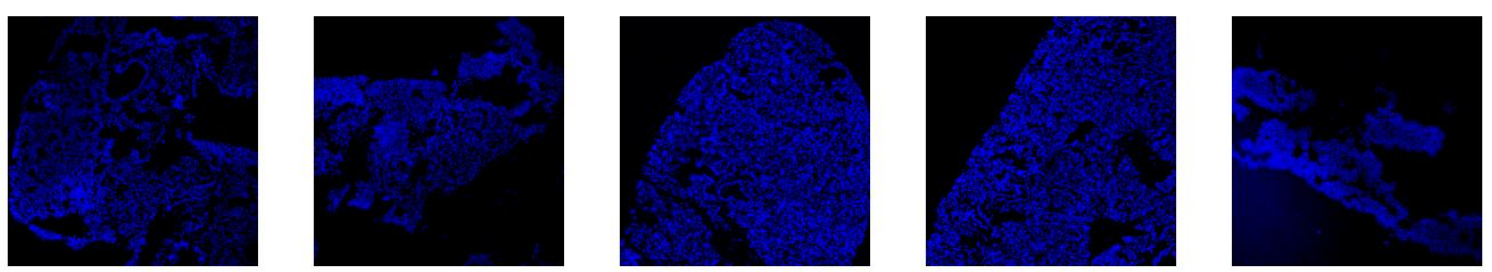

Day 6 Middle lobe of right lung
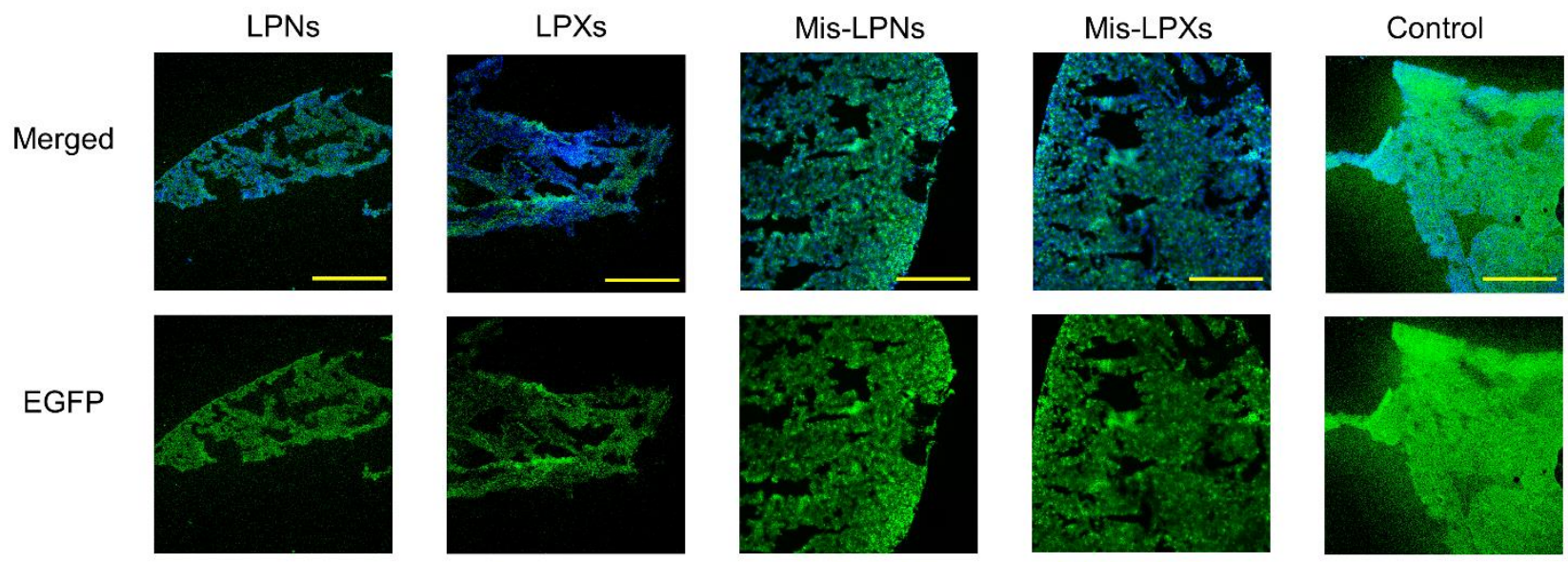

DAPI
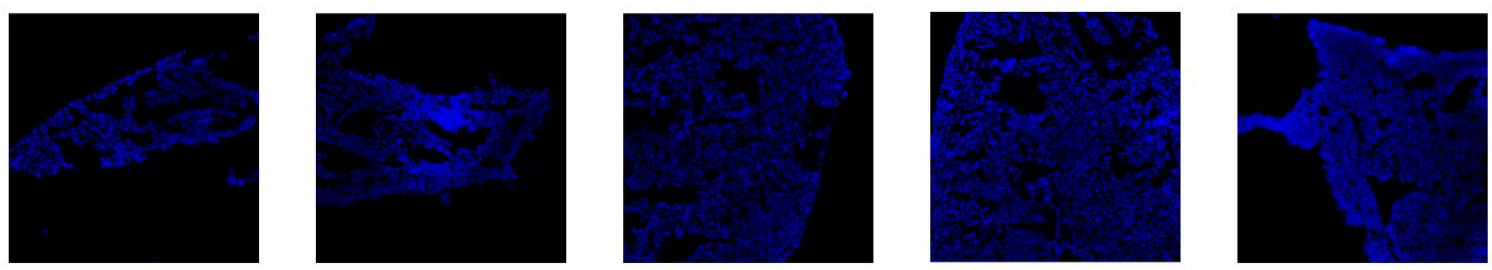

Day 6 Inferior lobe of right lung 

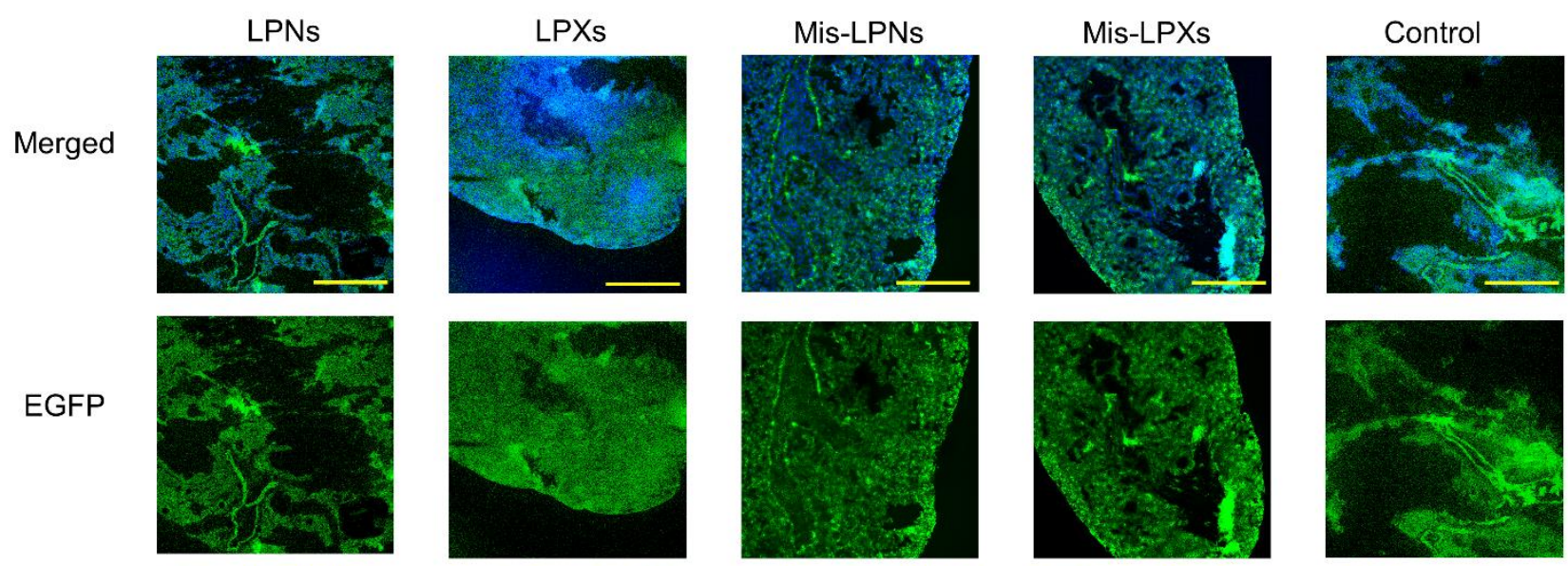

DAPI
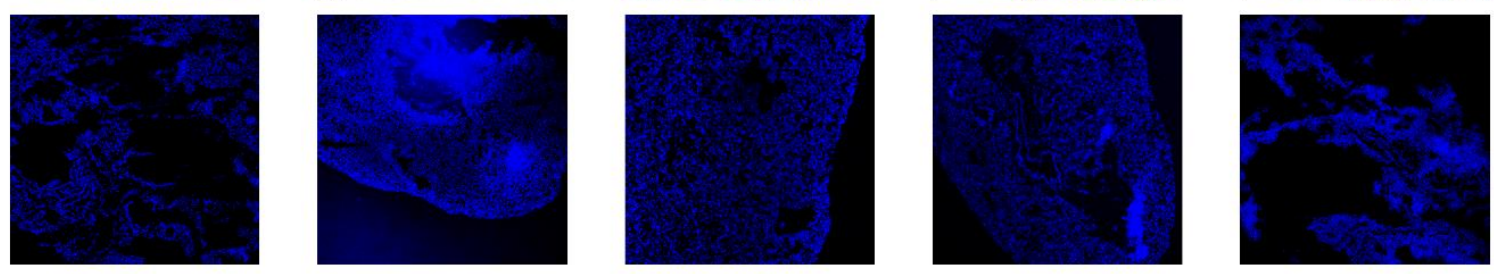

Day 6 Post-caval lobe

LPNs

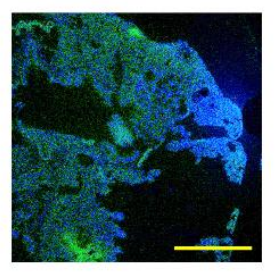

Merged

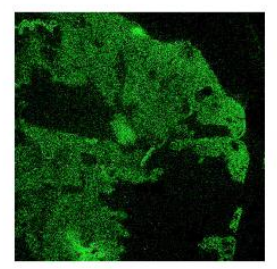

DAPI

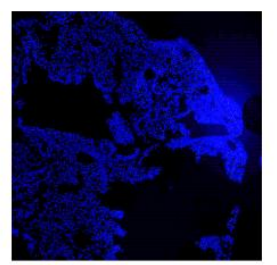

LPXs
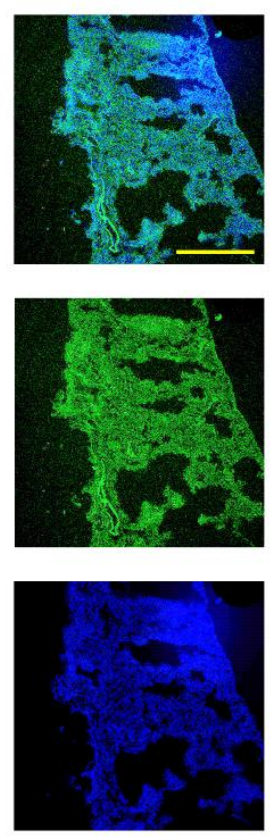
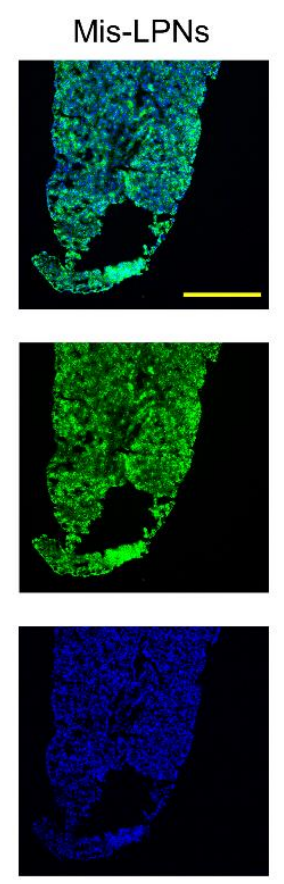

Day 11 Left lung
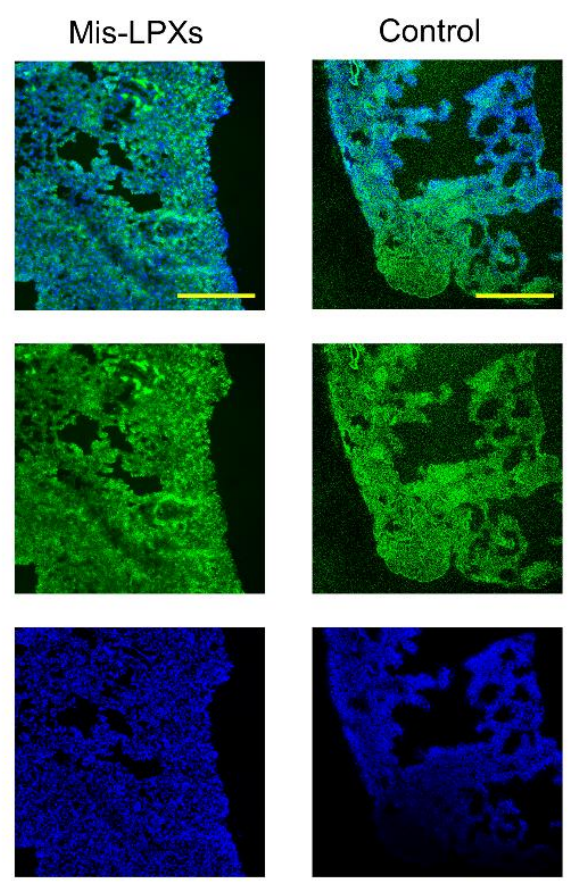

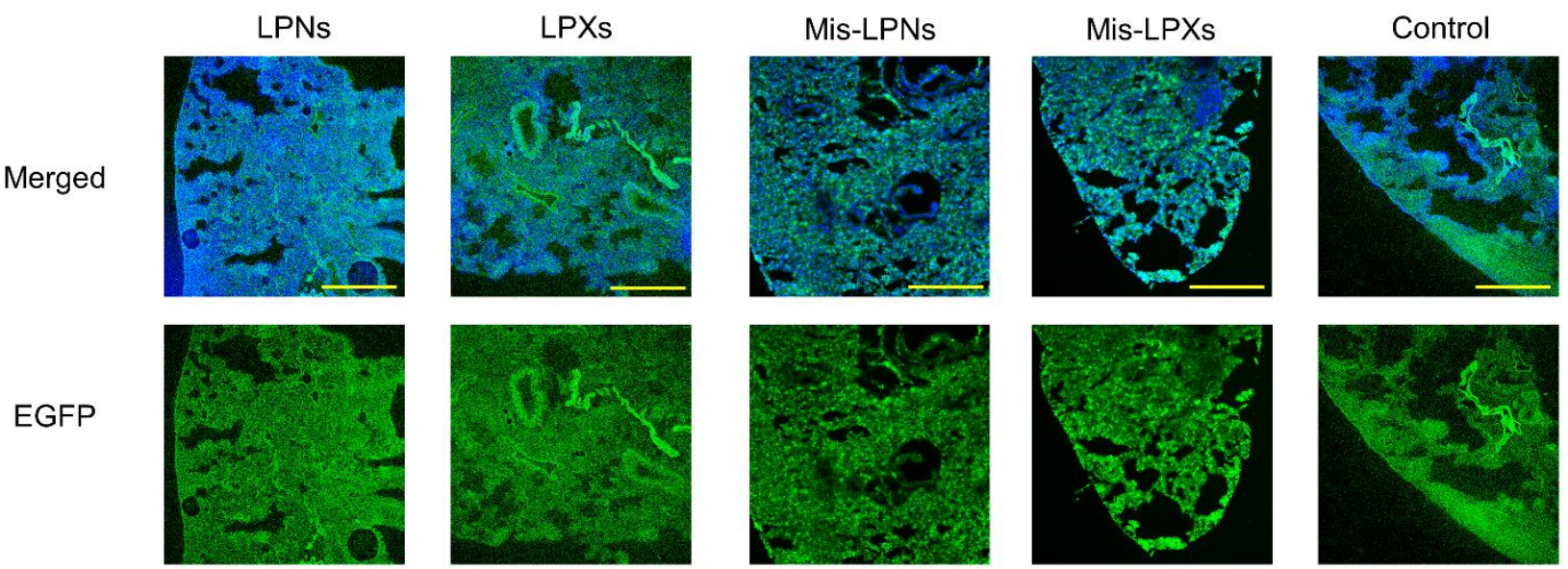

DAPI
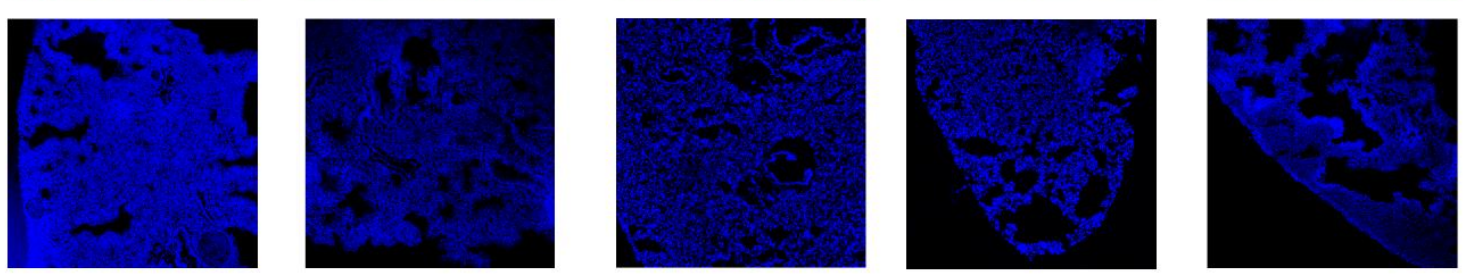

Day 11 Superior lobe of right lung
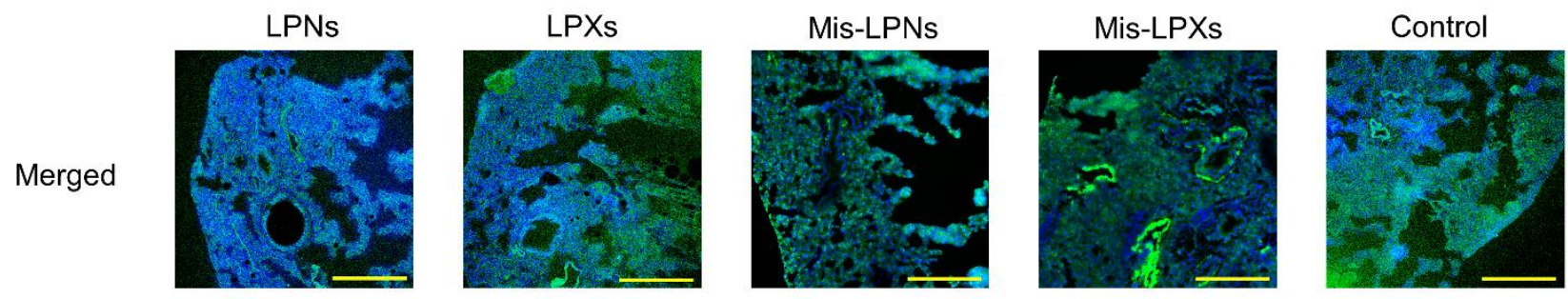

EGFP
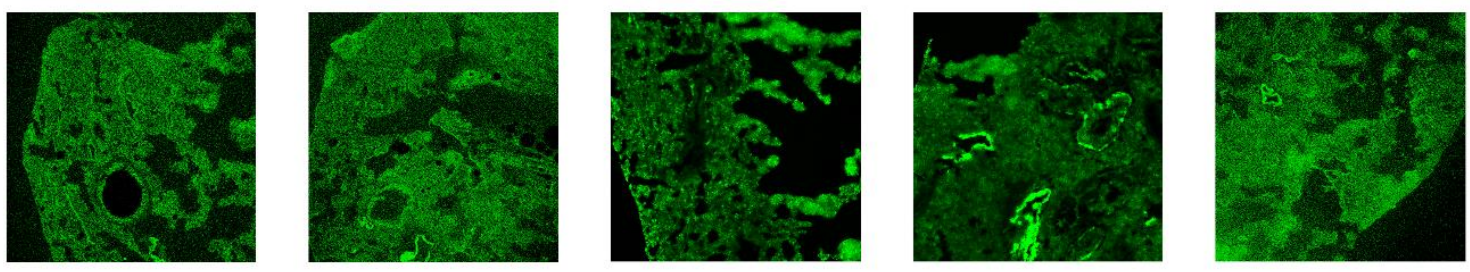

DAPI
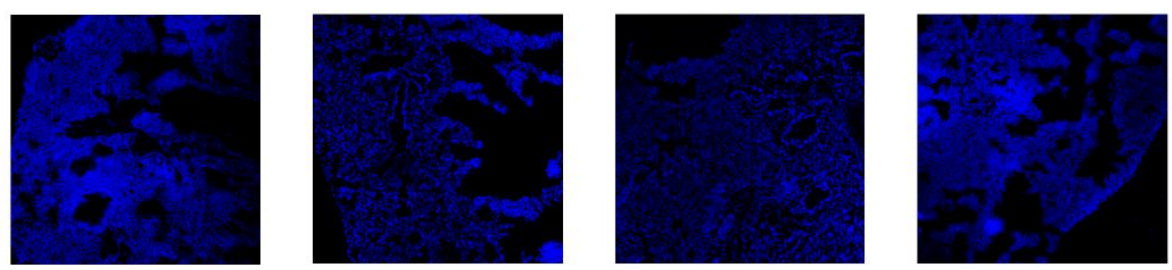

Day 11 Middle lobe of right lung 

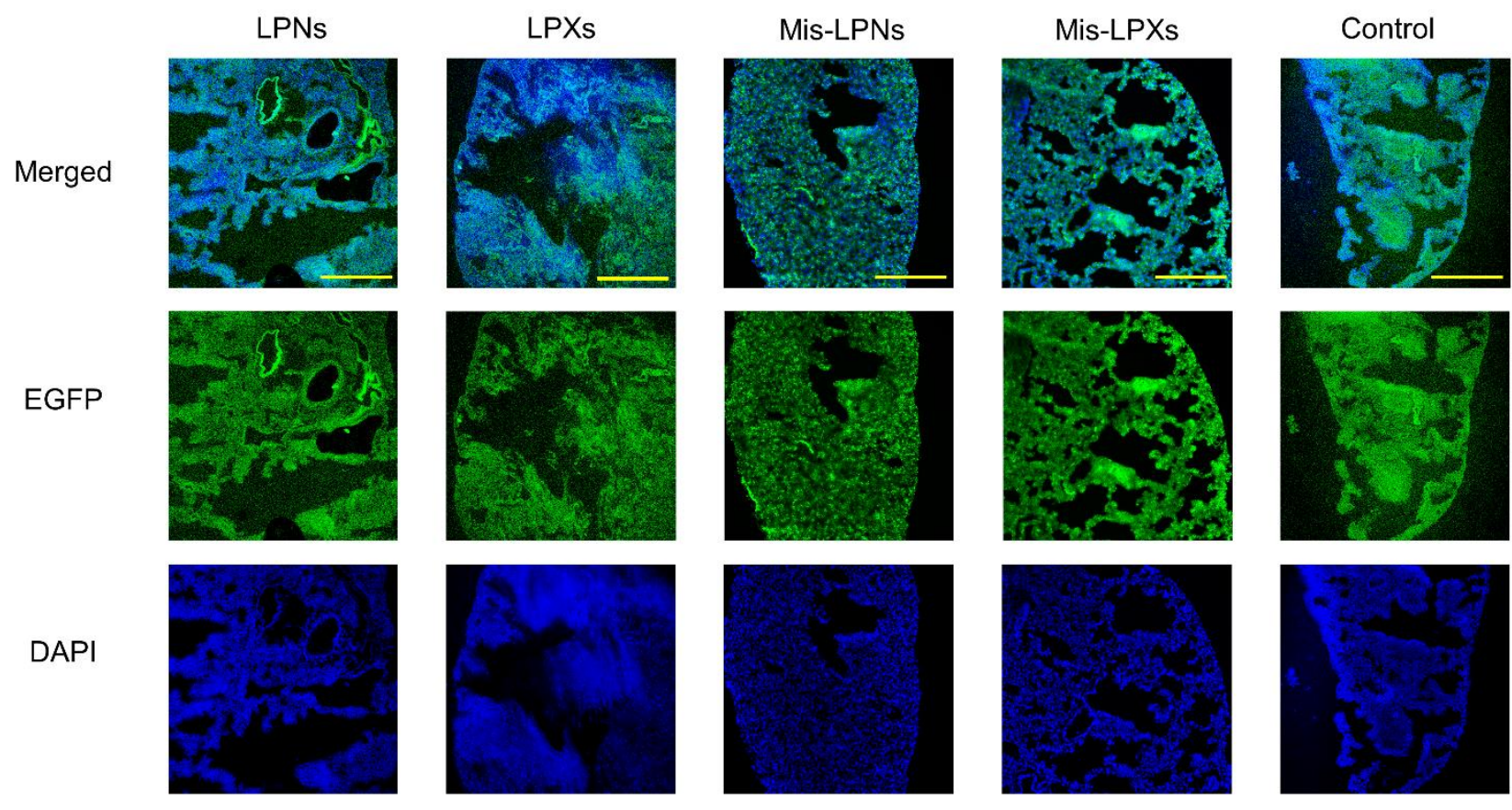

Day 11 Inferior lobe of right lung
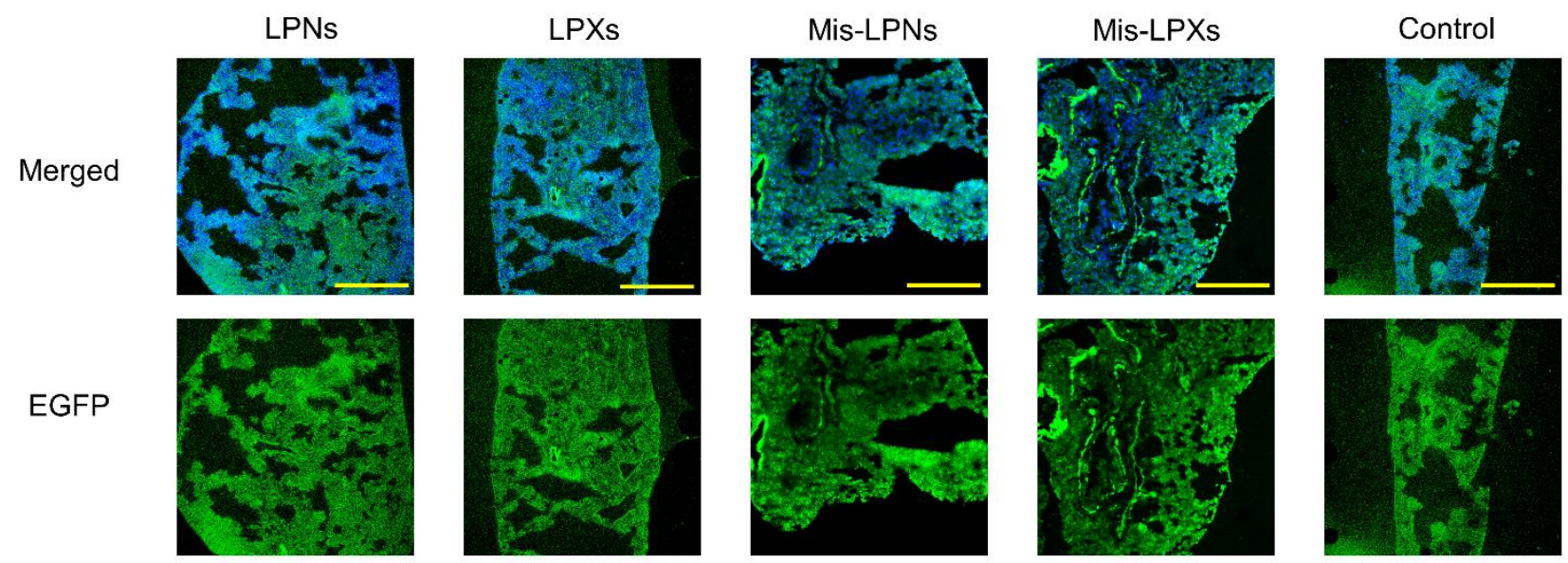

DAPI
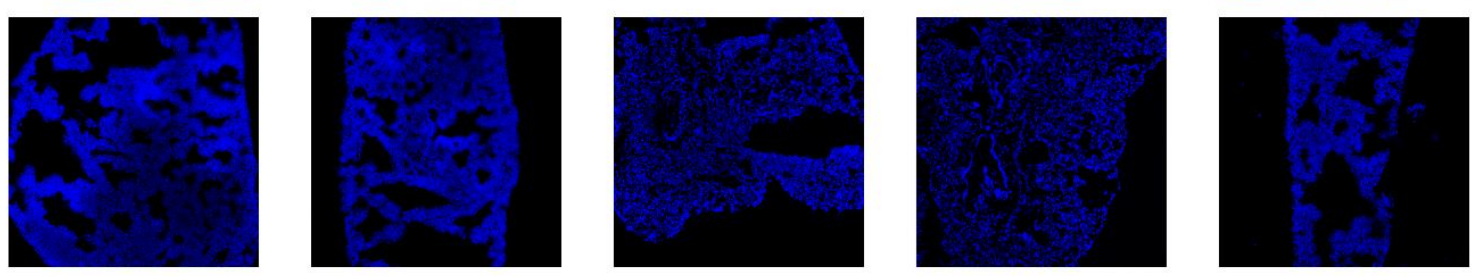

Day 11 Post-caval lobe

432 Figure S11. Representative CLSM images of lung tissue sections (scale bar $=200 \mu \mathrm{m}$ ).

434 The radiolabeling efficiencies of ${ }^{111}$ In to the LPNs and LPXs were $98.6 \%$ and $84.9 \%$, respectively, as 
shown by ITLC, where the radiolabeled nanoparticles stayed at $\mathrm{R}_{\mathrm{f}}=0$, while free ${ }^{111} \mathrm{In}^{3+}$ was seen at 436 the solvent front $\left(\mathrm{R}_{\mathrm{f}}=1\right)$ (Figure $\left.\mathrm{S} 12\right)$. The radiolabeling process had negligible effects on the 437 particle size, PDI and zeta potential of the two formulations (Table S2). There was no significant 438 difference before and after the radiolabeling.

439 Table S2. Particle size and zeta potential of nanoparticles before and after being radiolabeled (mean $440 \pm \mathrm{SD}, \mathrm{N}=3, \mathrm{n}=3)$.

\begin{tabular}{lllll}
\hline & Code & $\begin{array}{l}\text { Particle size } \\
(\mathbf{n m})\end{array}$ & PDI & $\begin{array}{l}\text { Zeta potential } \\
(\mathbf{m V})\end{array}$ \\
\hline \multirow{2}{*}{ LPNs } & Before radiolabeling & $239.3 \pm 1.5$ & $0.12 \pm 0.01$ & $34.2 \pm 0.7$ \\
& After radiolabeling & $235.9 \pm 2.1$ & $0.11 \pm 0.02$ & $32.4 \pm 1.0$ \\
& Before radiolabeling & $166.5 \pm 10.2$ & $0.22 \pm 0.01$ & $46.7 \pm 3.9$ \\
LPXs & After radiolabeling & $168.7 \pm 9.4$ & $0.20 \pm 0.06$ & $43.2 \pm 4.8$ \\
\hline
\end{tabular}

$$
\mathrm{R}_{\mathrm{f}}=1
$$<smiles>C[As][Te]C</smiles>
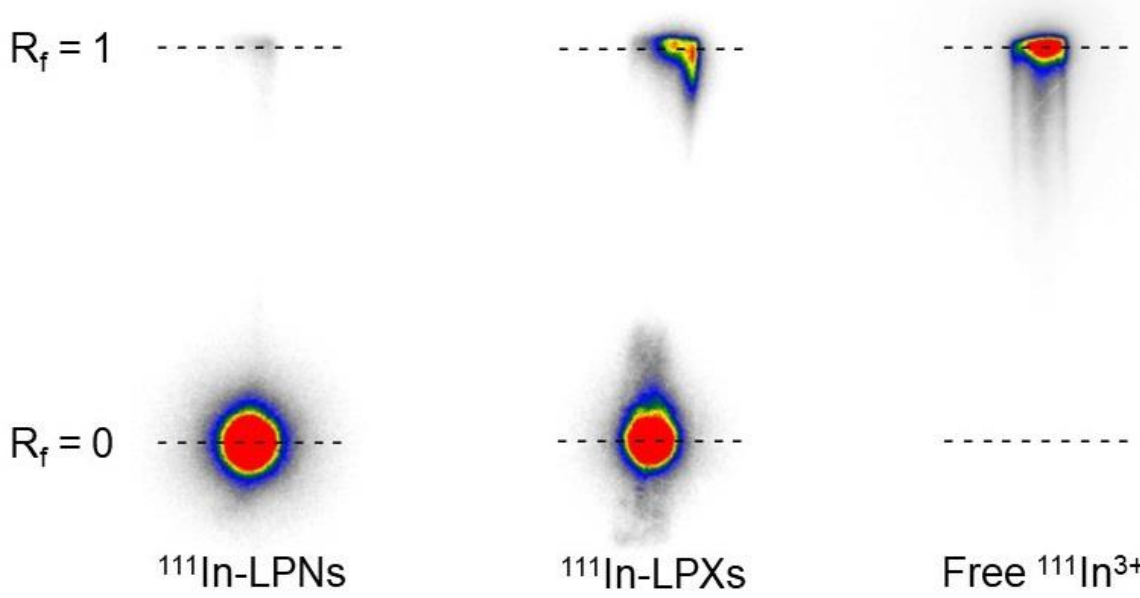

442 Figure S12. ITLC image of ${ }^{111}$ In radiolabeled nanoparticles using 0.1 M EDTA as the solvent.

\section{Endotoxin test}

444 The max endotoxin level of LPNs and LPXs suspension were $0.3 \pm 0.1 \mathrm{EU}$ and $0.5 \pm 0.3 \mathrm{EU}$, 445 respectively. The allowed endotoxin level was $2.5 \mathrm{EU}$ according to the Chinese Pharmacopeia (part 3, 446 1143, Edition 2015). 
448 The administration of LPXs resulted in an increase in IL-1 $\beta$ and IFN- $\gamma$ in BALF by $\mathrm{t}=72 \mathrm{~h}$, and 449 IL-4 level by $\mathrm{t}=6 \mathrm{~h}$. The administration of LPNs resulted in an increase in IL-1 $\beta$ at $\mathrm{t}=72 \mathrm{~h}$ but not 450 at other sampling points. Similar with LPNs, the administration of PNs did not result in any increase 451 in any sampling points and any cytokines tested in this study.

452 As for the total protein in BALF, the administration of LPXs resulted in an increase in the total protein level by 11 days (Figure S13D). Post-administration of LPNs resulted in an increase in BALF total protein at $\mathrm{t}=72 \mathrm{~h}$ and 11 days, while post-administration of PNs resulted in an increase in BALF total protein at $\mathrm{t}=24 \mathrm{~h}$ and $72 \mathrm{~h}$.
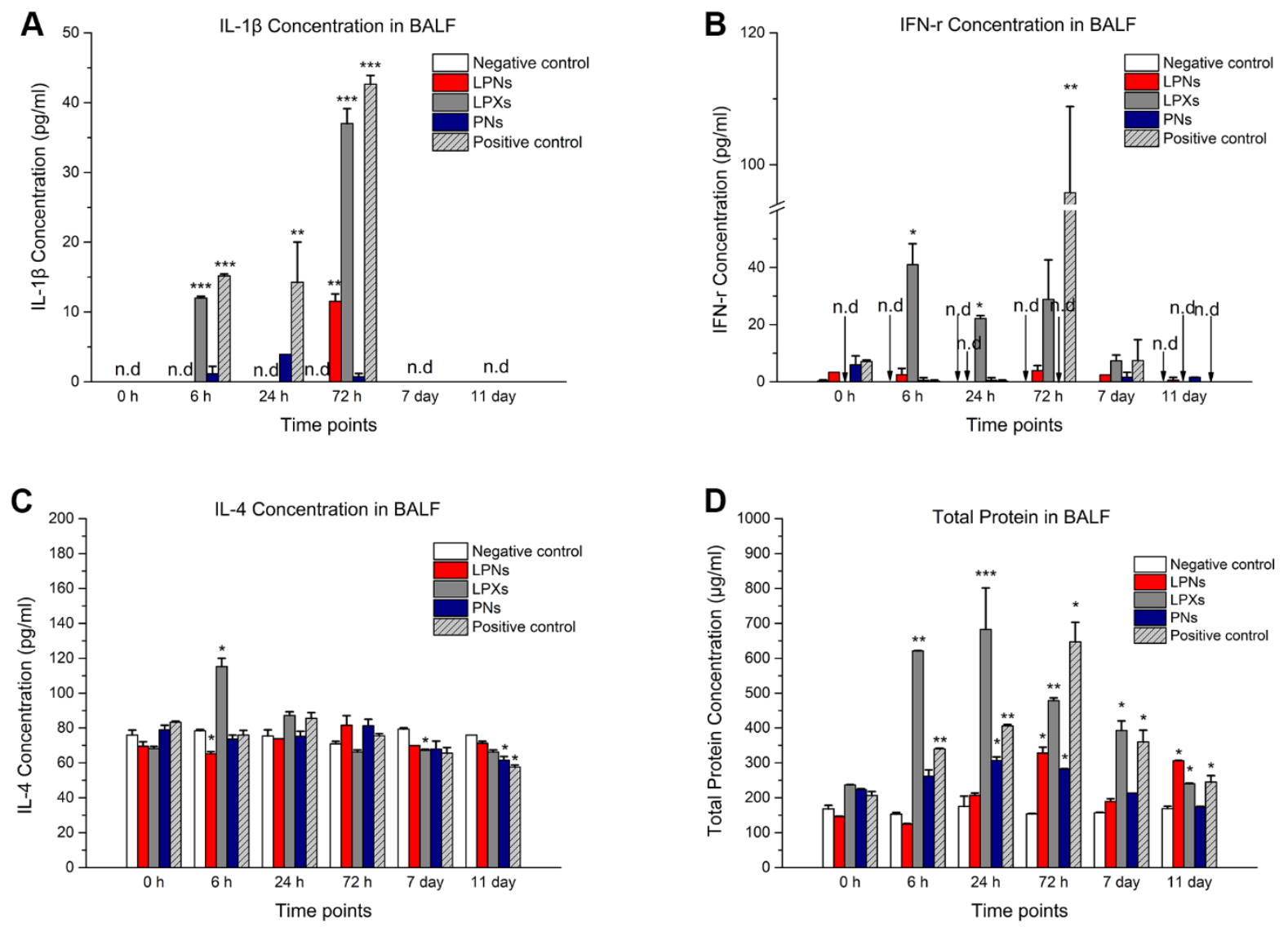

Figure S13. Assessment of cytokine levels and total protein in BALF. IL-1 $\beta$ (A), IFN- $\gamma(\mathrm{B})$, IL-4 (C), and total protein level in BALF (D). Significant difference in the inflammatory parameters compared 
459 with the respective PBS treated negative control: $* \mathrm{P} \leqslant 0.05, * * \mathrm{P} \leqslant 0.01, * * * \mathrm{P} \leqslant 0.001$. n.d., not 460 detectable, in case which the cytokine level was below the limit of detection of kits.

$461 \quad$ References

462 (1) Colombo, S., Cun, D., Remaut, K., Bunker, M., Zhang, J., Martin-Bertelsen, B., Yaghmur, A., 463 Braeckmans, K., Nielsen, H.M., Foged, C., Mechanistic Profiling of the Sirna Delivery Dynamics of 464 Lipid-Polymer Hybrid Nanoparticles, J Control Release, 2015, 201, 22-31.

465 (2) Marques, M., Löbenberg, R., Almukainzi, M., Simulated Biological Fluids with Possible 466 Application in Dissolution Testing, Dissolut Technol, 2011, 18, 15-28.

467 (3) Lauer, M.E., Dweik, R.A., Garantziotis, S., Aronica, M.A., The Rise and Fall of Hyaluronan in 468 Respiratory Diseases, Int J Cell Biol, 2015, 2015, 712507. 